\title{
The Homeodomain Transcription Factor NKX2.1 Is Essential for the Early Specification of Melanocortin Neuron Identity and Activates Pomc Expression in the Developing Hypothalamus
}

\author{
Daniela P. Orquera, ${ }^{1 *}$ M. Belén Tavella, ${ }^{1 *}$ Flavio S.J. de Souza, ${ }^{1,2,3}$ Sofía Nasif, ${ }^{1}{ }^{\oplus M a l c o l m ~ J . ~ L o w, ~}{ }^{4}$ \\ and ${ }^{\circ}$ Marcelo Rubinstein ${ }^{1,2,4}$ \\ ${ }^{1}$ Instituto de Investigaciones en Ingeniería Genética y Biología Molecular, Consejo Nacional de Investigaciones Científicas y Técnicas, 1428 Buenos Aires, \\ Argentina, ${ }^{2}$ Departamento de Fisiología, Biología Molecular y Celular, Facultad de Ciencias Exactas y Naturales, Universidad de Buenos Aires, 1428 Buenos \\ Aires, Argentina, ${ }^{3}$ Instituto de Fisiología, Biología Molecular y Neurociencias, Universidad de Buenos Aires y Consejo Nacional de Investigaciones Científicas y \\ Técnicas, 1428 Buenos Aires, Argentina, and ${ }^{4}$ Department of Molecular and Integrative Physiology, University of Michigan, Ann Arbor, Michigan 48105
}

Food intake is tightly regulated by a group of neurons present in the arcuate nucleus of the hypothalamus, which release Pomc-encoded melanocortins, the absence of which induces marked hyperphagia and early-onset obesity. Although the relevance of hypothalamic POMC neurons in the regulation of body weight and energy balance is well appreciated, little is known about the transcription factors that establish the melanocortin neuron identity during brain development and its phenotypic maintenance in postnatal life. Here, we report that the transcription factor NKX2.1 is present in mouse hypothalamic POMC neurons from early development to adulthood. Electromobility shift assays showed that NKX2.1 binds in vitro to NKX binding motifs present in the neuronal Pomc enhancers nPE1 and nPE2 and chromatin immunoprecipitation assays detected in vivo binding of NKX2.1 to nPE1 and nPE2 in mouse hypothalamic extracts. Transgenic and mutant studies performed in mouse embryos of either sex and adult males showed that the NKX motifs present in nPE1 and nPE2 are essential for their transcriptional enhancer activity. The conditional early inactivation of $N k x 2.1$ in the ventral hypothalamus prevented the onset of Pomc expression. Selective Nkx2.1 ablation from POMC neurons decreased Pomc expression in adult males and mildly increased their body weight and adiposity. Our results demonstrate that NKX2.1 is necessary to activate Pomc expression by binding to conserved canonical NKX motifs present in nPE1 and nPE2. Therefore, NKX2.1 plays a critical role in the early establishment of hypothalamic melanocortin neuron identity and participates in the maintenance of Pomc expression levels during adulthood.

Key words: body weight regulation; conditional mutant mice; melanocortins; neuron-specific expression; transcription factor

Significance Statement

Food intake and body weight regulation depend on hypothalamic neurons that release satiety-inducing neuropeptides, known as melanocortins. Central melanocortins are encoded by Pomc, and Pomc mutations may lead to hyperphagia and severe obesity. Although the importance of central melanocortins is well appreciated, the genetic program that establishes and maintains fully functional POMC neurons remains to be explored. Here, we combined molecular, genetic, developmental, and functional studies that led to the discovery of NKX2.1, a transcription factor that participates in the early morphogenesis of the developing hypothalamus, as a key player in establishing the early identity of melanocortin neurons by activating Pomc expression. Thus, Nkx2.1 adds to the growing list of genes that participate in body weight regulation and adiposity.

\section{Introduction}

The arcuate nucleus of the hypothalamus is a brain hub that integrates nutritional and hormonal information to promote food intake or satiety (Rubinstein and Low, 2017). A group of

Received Nov. 15, 2018; revised March 3, 2019; accepted March 8, 2019.

Author contributions: D.P.O., M.B.T., F.S.J.d.S., S.N., M.J.L., and M.R. designed research;D.P.O., M.B.T., F.S.J.d.S.,

S.N., and M.R. performed research; M.J.L. and M.R. contributed unpublished reagents/analytic tools; D.P.O., M.B.T.,

F.S.J.d.S., S.N., and M.R. analyzed data; M.R. wrote the paper. arcuate neurons expresses proopiomelanocortin (Pomc), a gene that encodes the anorexigenic neuropeptides $\alpha-, \beta$-, and $\gamma$ melanocyte-stimulating hormone, collectively known as central melanocortins (Rubinstein and Low, 2017). POMC neurons pro-
This work was supported by National Institutes of Health Grant R01-DK-068400 (to M.J.L. and M.R.), Agencia Nacional de Promoción Científica y Tecnológica (to M.R.) and UBACYT (M.R.). We thank Marta Treimun for technical assistance in mice production, Dr. Shioko Kimura for providing the Nkx2.1 conditional mutant mice, and Lucía Franchini for valuable protocols and ideas. 
mote satiety upon sensing variations in glucose (Ibrahim et al., 2003), leptin (Cowley et al., 2001), and insulin (Benoit et al., 2002) levels as well as in local temperature (Jeong et al., 2018). In addition, POMC neurons receive multiple synaptic connections from a variety of neurons that, together, orchestrate food intake (Heisler et al., 2002; A ponte et al., 2011; Chen et al., 2015; Jeong et al., 2017; Rau and Hentges, 2017). The physiological importance of central melanocortins is evident in hypothalamic Pomcdeficient mice, which are hyperphagic and display early-onset extreme obesity (Bumaschny et al., 2012; Chhabra et al., 2016). In addition, humans (Krude et al., 1998) and mice (Yaswen et al., 1999) carrying null allele mutations in POMC or in the melanocortin receptor 4 (Huszar et al., 1997; Farooqi et al., 2000) are also hyperphagic and severely obese. Although POMC neurons play a fundamental role in the regulation of food intake and body weight, little is known about the genetic programs that establish their identity and phenotypic maintenance.

Hypothalamic Pomc expression is controlled by two upstream distal enhancers, nPE1 and nPE2, which are highly conserved in mammals (de Souza et al., 2005). Although nPE1 and nPE2 have unrelated evolutionary origins, both enhancers drive overlapping spatiotemporal activities to the entire population of hypothalamic POMC neurons (Santangelo et al., 2007; Franchini et al., 2011). Targeted mutagenesis of nPE1 and/or nPE2 revealed their partially redundant enhancer function and cooperativity to maintain Pomc levels above a critical functional threshold (Lam et al., 2015). Interestingly, only the concurrent removal of both enhancers reduced Pomc expression to very low levels, leading to hyperphagia and early-onset obesity (Lam et al., 2015). Because nPE1 and nPE2 act as transcriptional Pomc enhancers in the same population of hypothalamic neurons, it is conceivable that they share DNA elements that recruit similar transcription factors (TFs). In fact, we detected a 21 bp sequence embedded in a highly conserved region of nPE1 and a similar sequence in nPE2 (Lam et al., 2015), which contain ATTA motifs typically recognized by homeodomain TFs (Lam et al., 2015). Recently, we found that these motifs are recognized by Islet 1 (ISL1), a LIM-homeodomain TF that coexpresses with Pomc in the developing hypothalamus and postnatal life (Nasif et al., 2015). Moreover, early inactivation of Isl1 prevents the onset of hypothalamic Pomc expression, and the ablation of Isl1 from POMC neurons impairs Pomc expression in adult mice and leads to hyperphagia and obesity (Nasif et al., 2015).

Although ISL1 is necessary for Pomc expression, it is clearly not sufficient. Isll is expressed not only in a much broader hypothalamic territory than Pomc, but also in other neuronal types where Pomc is never found (Ehrman et al., 2013; Mazzoni et al., 2013; Cho et al., 2014). Thus, other TFs are likely to participate in the arcuate-specific expression of Pomc. By combining molecular, cellular, genetic, and functional studies, we demonstrate here that the homeodomain TF NKX2.1 activates arcuate Pomc expression from early development to adulthood by interacting with motifs present in nPE1 and nPE2. Therefore, NKX2.1 defines the identity of hypothalamic melanocortin neurons and is necessary to maintain normal levels of arcuate Pomc mRNA and body weight regulation in adult mice.

*D.P.O. and M.B.T. contributed equally to this work.

The authors declare no competing financial interests.

Correspondence should be addressed to Marcelo Rubinstein at mrubins@dna.uba.ar.

https://doi.org/10.1523/JNEUROSCI.2924-18.2019

Copyright $(2019$ the authors

\section{Materials and Methods}

Breeding of mice. Mice of both sexes were housed in ventilated cages under controlled temperature and photoperiod (12 h light/dark cycle, lights on from 7:00 A.M. to 7:00 P.M.), and fed ad libitum. All procedures followed the Guide for the Care and Use of Laboratory Animals and performed in agreement with the Instituto de Investigaciones en Ingeniería Genética y Biología Molecular "Dr. Héctor N. Torres"-CONICET institutional animal care and use committee. $N k \times 2.1^{\text {loxP/loxP }}$ mice were provided by Dr. Shioko Kimura; (NIH, Bethesda, Maryland) and maintained as homozygotes on a C57BL/6J background. CAAG-CreERT mice (Hayashi and McMahon, 2002) were obtained from The Jackson Laboratory (B6.Cg-Tg[CAG-Cre/Esr1]5Amc/J) and were intercrossed with $N k x 2.1^{\text {loxP/loxP }}$ mice to generate the Ind Nkx2.1 KO strain. Isl1-Cre (Yang et al., 2006) mice were obtained from The Jackson Laboratory ( Isli $\left.{ }^{\text {tml } 1(\mathrm{cre}) S e v} / \mathrm{J}\right)$ and maintained as heterozygotes on a C57BL/6J background. Genotyping was performed by PCR using genomic DNA extracted from ear biopsy samples or embryo fragments. All primer sequences used are available in Table 1.

Transgene and transgenic mice production. Transgene nPE2PomcEGFP was previously reported (de Souza et al., 2005; Franchini et al., 2011). Transgene $\mathrm{nPE} 2\left(\mathrm{NKX}^{\star}\right)$ Pomc-EGFP is identical except for carrying two transversion mutations in each of the two NKX binding sites present in nPE2 (TCAAG $\rightarrow$ TCCCG and TCAAT $\rightarrow$ TCCCT). This transgene was generated using a standard megaprimer PCR protocol as previously described (Nasif et al., 2015) but with the primers described in Table 1. Introduction of mutations was confirmed by sequencing. Transgenes were digested with NotI and SalI restriction enzymes and the fragment of interest was separated and extracted from a $0.8 \%$ agarose-TBE (Tris-borate-EDTA) gel with QIAquick Gel Extraction Kit (catalog \#28704, Qiagen). The transgene was further concentrated and purified with DNA Clean \& Concentrator-5 (catalog \#D4014, Zymo Research) and eluted in $5 \mu \mathrm{l}$ of EmbryoMax Injection Buffer (catalog \#MR-095$10 \mathrm{~F}$, Millipore). Transgenic mice were produced by pronuclear microinjection of the transgene in FVB zygotes. Transgenic pups were identified by PCR using M329 and M330 primers (Table 1). nPE2 was amplified using $\Delta 2.3$ and $\Delta 2.5$ primers (Table 1), cloned using pGEM-T Easy Vector System I (catalog \#A1360, Promega) and sequenced to confirm mutations. Founder mice were intercrossed with FVB wild-type mice, and F1 newborns were analyzed.

Knock-out mice generation. The CRISPR (clustered regularly interspaced short palindromic repeats)/Cas9 system was used to generate deletions encompassing the NKX binding site in nPE1. A CRISPR guide was selected using the website of the Zhang laboratory (crispr.mit.edu). Guide CCCCACAATGGGGCTTGAAG was selected because it falls next to the NKX binding site and had a high-quality score and thus low chances of producing off-target mutations. The guide was subcloned in plasmid DR274 (Plasmid \#42250, Addgene), and single guide RNA (sgRNA) was synthesized using MEGAshortscript T7 Transcription Kit (catalog \#AM1354, Ambion). Cas9 mRNA was synthesized from plasmid MLM3613 (Plasmid \#42251, Addgene) using mMESSAGE mMACHINE T7 Transcription Kit (catalog \#AM1344, Ambion) and Poly(A) Tailing Kit (catalog \#AM1350, Ambion). Cas9 mRNA (50 ng/ $\mu \mathrm{l})$ and sgRNA $(50 \mathrm{ng} / \mu \mathrm{l})$ were injected in the cytoplasm of Pomc ${ }^{+/ \Delta \mathrm{nPE} 2}$ C57BL/6J zygotes. Founder mice were analyzed by PCR using primers available in Table 1 and PCR products subcloned in pGEM.T Easy Vector for sequencing. A strain carrying a 34 bp deletion (chr12:3,942,178-3,942,211) in $\mathrm{nPE} 1$ in a $\triangle \mathrm{nPE} 2$ allele was selected and maintained in a C57BL/6J background and F2 mice were analyzed.

Tamoxifen injections. Tamoxifen (TAM; catalog \#T5648, SigmaAldrich) was dissolved in sesame oil (catalog \#S3547, Sigma-Aldrich) at $15 \mathrm{mg} / \mathrm{ml}$ by sonication. One milliliter aliquots were stored at $-20^{\circ} \mathrm{C}$ and thawed for $10 \mathrm{~min}$ at $37^{\circ} \mathrm{C}$ before injection. Pregnant females were injected intraperitoneally with a dose of $133 \mathrm{mg} / \mathrm{kg}$.

Tissue collection and embedding. Pregnant mice at $10.5-15.5 \mathrm{~d}$ postcoitum (dpc; the day of detection of vaginal plug was considered dpc 0.5 ) were dislocated and embryos of either sex were collected and washed in cold RNase-free PBS. Fixation was performed with $4 \%$ PFA-PBS at $4^{\circ} \mathrm{C}$, for a period of time dependent on developmental stage. The 10.5, 11.5, 


\section{Table 1. Primer sequences used to detect different alleles and mutations and probes for EMSA studies}

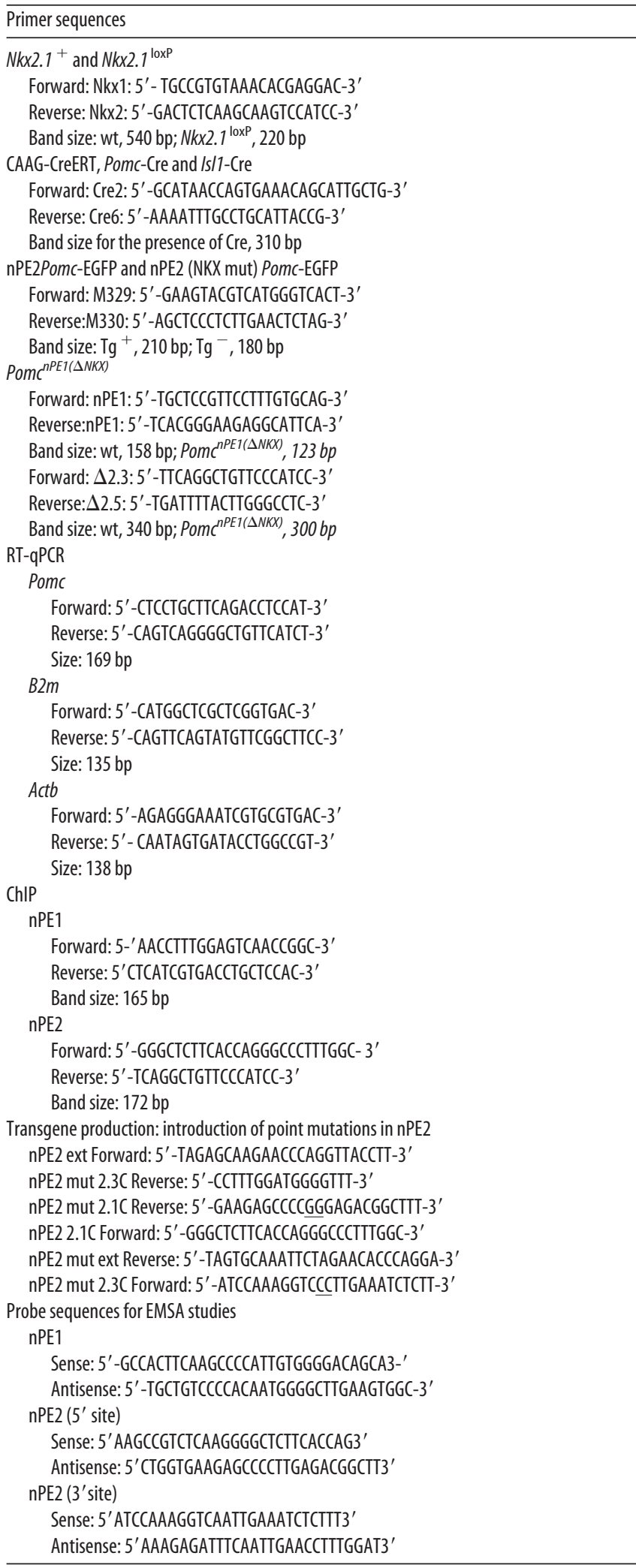

and $12.5 \mathrm{dpc}$ embryos were fixed for $2 \mathrm{~h}$, the heads of $15.5 \mathrm{dpc}$ embryos were dissected and fixed for $4 \mathrm{~h}$, and the heads of newborns were fixed overnight. Samples were then washed in cold PBS and cryoprotected with $10 \%$ sucrose-PBS overnight. Tails or posterior limbs were cut to obtain tissue for genotyping. Tissues were then stabilized in $10 \%$ sucrose- $10 \%$
gelatin-PBS at $37^{\circ} \mathrm{C}$ for $30 \mathrm{~min}$ before freezing. Adult mice of either sex were perfused using $4 \%$ PFA, and brains were collected and fixed overnight in $4 \%$ PFA at $4^{\circ} \mathrm{C}$. Whole brains were then washed in cold PBS and cryoprotected with $10 \%$ sucrose-PBS overnight. Hypothalamic sections were then cut grossly using a metallic adult brain mold before freezing. Gelatin blocks containing the tissues were stuck on cork sheets with Tissue-Tek OCT compound (catalog \#4583, Sakura Finetek). Gelatin blocks were snap-frozen in 2-methylbutane (catalog \#M32631, SigmaAldrich) at $-55^{\circ} \mathrm{C}$ and stored at $-80^{\circ} \mathrm{C}$ for up to 1 year. The $20 \mu \mathrm{m}$ slices were cut in a cryostat (CM 1850, Leica) and mounted on microscope slides (catalog \#12-550-15, Superfrost Plus, Fisherbrand). Sections were then air dried overnight and stored at $-20^{\circ} \mathrm{C}$ until use.

Immunofluorescence and cell quantification. Immunofluorescence was performed as described previously (Nasif et al., 2015). Primary antibodies used were as follows: rabbit anti-rACTH (repository adrenocorticotropic hormone; 1:1000; National Hormone and Peptide Program); mouse anti-ISL1 (1:20; catalog \#40.2D6, Developmental Studies Hybridoma Bank, University of Iowa); rabbit anti-NKX2.1 (1:1000; catalog \#ab40880, Abcam); rabbit anti-NKX2.1 (1:1000; catalog \#sc-13040, Santa Cruz Biotechnology); chicken anti-EGFP (1:2000; GFP-1020, Aves Labs); mouse anti-ASCL1 (1:200; catalog \#556604, BD PharMingen); mouse anti-NGN3 (1:2000; catalog \#F25A1B3, Developmental Studies Hybridoma Bank, University of Iowa); and rabbit anti-NPY (1:1000; T-4070 Peninsula Laboratories). Secondary antibodies were anti-mouse or anti-rabbit Alexa Fluor 555 (1:1000; Invitrogen); anti-mouse, antirabbit Alexa Fluor 488 (1:1000; Invitrogen); or anti-chicken Alexa Fluor 488 (1:2000; catalog \#703-545-155, Jackson ImmunoResearch). Depending on the marker antigen, immunohistochemistry experiments were repeated in two to five different embryos, with consistent results. Nuclei were stained with a $1 \mathrm{mg} / \mathrm{L}$ dilution of DAPI for $10 \mathrm{~min}$ and then washed twice before mounting with Vectashield (Vector Labs). Confocal images for coexpression assays and cell counting were obtained using a FV300 system combined with a BX51 Microscope (Olympus) or a Confocal TCS-SPE Microscope (Leica). The number of $\mathrm{EGFP}^{+}$, fluorescently immunolabeled $\mathrm{ACTH}^{+}$, or ISL1 ${ }^{+}$cells were counted by confocal microscopy. For embryonic day 11.5 (E11.5) and E12.5 embryos, we collected sagittal $20 \mu \mathrm{m}$ sections spanning the whole hypothalamus and $\mathrm{POMC}^{+}$ or $\mathrm{NPY}^{+}$cells counted manually in anatomically similar sections. The number of $\mathrm{ACTH}^{+}$cells in E15.5 embryos was calculated automatically using Image $(\mathrm{NIH})$ by averaging two symmetrical sections. In adult mice, $P$ omc-EGFP ${ }^{+}$cells were counted manually in two coronal slices at the median eminence level and averaged. Quantitative densitometry of NPY-labeled neurons was calculated using ImageJ in a sagittal section for E15.5 embryos and two coronal slices at the median eminence level for adults, and averaged. At least three animals were used for each determination.

Pomc $m R N A$ quantification by quantitative real-time PCR. Whole male mouse adult hypothalami or embryo heads of either sex were dissected, collected in ice-cold TriPure Isolation Reagent (catalog \#11667165001, Sigma-Aldrich) and stored at $-80^{\circ} \mathrm{C}$ until RNA extraction, which was performed following manufacturer instructions. RNA integrity was assessed by gel electrophoresis; clear $28 \mathrm{~S}$ and $18 \mathrm{~S}$ rRNA were observed in an approximate 2:1 ratio. Quantification was performed using a Nanodrop and 260:280 and 260:230 ratios were checked to assess purity. One microgram of RNA was DNase I (catalog \#AM2222, Ambion) treated and used for first-strand cDNA synthesis, using the High Capacity Reverse Transcription Kit with random primers (catalog \#4368814, Applied Biosystems). Primers were designed with Primer 3 program. Pomc mRNA was quantified using primers spanning exons 2 and 3 relative to internal control genes $\beta 2$-microglobulin and $\beta$-actin. Primer sequences are listed in Table 1. Samples were run in triplicate in an Applied Biosystems 7500 Real-Time PCR System machine using Power Up SYBR Green Master Mix (catalog \#A25472, Applied Biosystems). Melt curves were analyzed to confirm specificity of PCR product. Relative quantification was performed by interpolating $\mathrm{Ct}$ values in standard curves or by $2-\Delta \Delta \mathrm{CT}$.

Electrophoretic mobility shift assay. A mouse Nkx2.1 cDNA clone (catalog \#10698306, ATCC) was subcloned into the expression vector pGEX $4 \mathrm{~T} 3$ using NcoI and EcoRI restriction enzymes and transformed into the Escherichia coli BL21 strain. Protocols for obtaining bacterial extracts, 

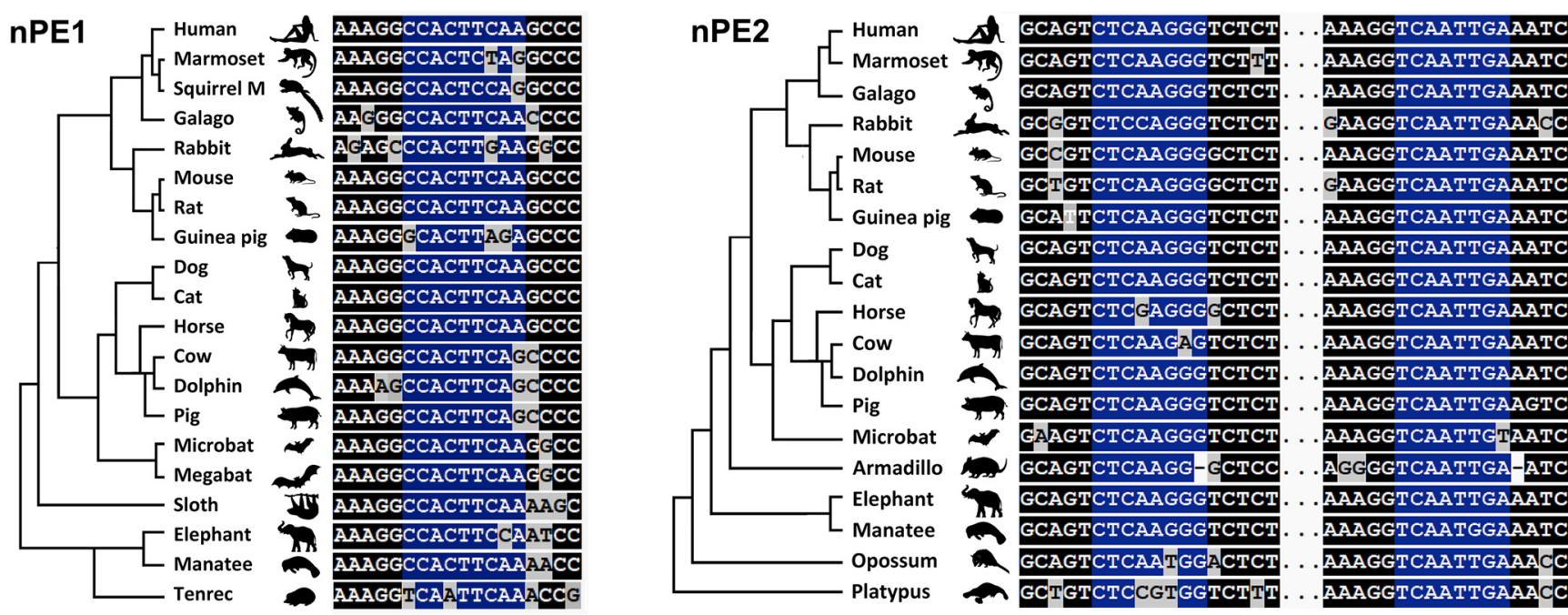

Figure 1. The Pome neuronal enhancers nPE1 and nPE2 contain canonical binding motifs for a transcription factor of the NKX family, which are highly conserved in all mammalian orders. Left, Evolutionary tree of placental mammals followed by a ClustalW alignment showing the unique NKX binding site in nPE1. Right, Evolutionary tree of mammals followed by a ClustalW alignment of the two NKX binding sites present in nPE2. Conserved nucleotides are indicated in a dark background, and those conserved nucleotides corresponding to canonical NKX binding sites in blue. See extended ClustalW for the entire nPE1 and nPE2 enhancers in Figure 1-1 (available at https://doi.org/10.1523/JNEUROSCI.2924-18.2019.f1-1) and Figure 1-2 (available at https://doi.org/10.1523/ JNEUROSCI.2924-18.2019.f1-2), respectively.

radioactive labeling of DNA probes, and preparation of electrophoretic mobility shift assay (EMSA) reactions were previously described (de Souza et al., 2011). After electrophoresis, gels were dried and exposed to $\mathrm{x}$-ray film (BioMax MS, Kodak) or Storage Phosphor Screen that was subsequently scanned in a STORM 860 Phospho Imager using ImageQuant5.2 software (GE Healthcare). Probe sequences are shown in Table 1.

Chromatin immunoprecipitation. Hypothalami from five adult mice were collected and fixed in $1 \%$ PFA-PBS for $20 \mathrm{~min}$ at $4^{\circ} \mathrm{C}$. Samples were sonicated to obtain 200-400 bp chromatin fragments. Forty micrograms of chromatin were immunoprecipitated with $5 \mu \mathrm{g}$ of NKX2.1 antibody (catalog \#sc-13040x, Santa Cruz Biotechnologies) or Normal Rabbit IgG (catalog \#12-370, Merck Millipore). After overnight incubation, antibody-chromatin complexes were pulled down using a mixture of salmon sperm DNA blocked Protein G PLUS-agarose and Protein A-agarose (catalog \#sc-2002, \#sc-2001, Santa Cruz Biotechnologies). Beads were washed with low salt (Tris-HCl pH 8, 10 mm, Triton X-100 1\%, EDTA 2 mm, SDS $0.1 \%, \mathrm{NaCl} 150 \mathrm{~mm}$ ), high salt (as low salt but with $\mathrm{NaCl} 450 \mathrm{~mm}$ ), $\mathrm{LiCl}$ [Tris-HCl pH:8 10 mm, LiCl 0.25 m, IGEPAL-CA630 1\%, EDTA 1 mM, Na deoxycholate $1 \%(\mathrm{w} / \mathrm{v})$ ] and TE (twice) buffers. Elution was performed overnight at $65^{\circ} \mathrm{C}$ with $10 \mu \mathrm{g}$ of Proteinase $\mathrm{K}$ in Tris- $\mathrm{HCl} \mathrm{pH} 810 \mathrm{~mm}$, SDS $1 \%$, and $\mathrm{NaCl} 340 \mathrm{~mm}$. DNA was purified with one volume of phenol: isoamyl alcohol: chloroform (25:1:24 v/v/v) followed by a volume of isoamyl alcohol:chloroform $(1: 24, \mathrm{v} / \mathrm{v})$ and then precipitated with sodium acetate and ethanol. Immunoprecipitated fragments were analyzed by end-point PCR (primer information is available in Table 1).

Statistics. All data presented are the mean \pm SEM and were analyzed using GraphPad Prism Software (version 5.01, 2007, GraphPad Software) or R Studio (version 1.1.456) by Student's $t$ test or ANOVA, except for cell counts. Post hoc pairwise comparisons between groups were performed by Tukey test. Normality of the distributions was assessed by Shapiro-Wilk test $(p>0.05)$, and the equality of the variance with the Bartlett's or $F$ test $(p>0.05)$. When Pomc mRNA was quantified in embryos, variance was unequal and VarPower function of "nmle" package in R Studio (Pinheiro et al., 2018) was used. Generalized linear models (GLMs; using "lme4" package, R studio) for Poisson or binomial distribution were used to assess the significance of difference in the number of cells. $p$ Values $<0.05$ were considered to be significant.

\section{Results}

NKX2.1 is a candidate TF for the regulation of hypothalamic Pomc expression

Within the neuronal Pomc enhancer nPE1, we detected a canonical binding motif for a TF of the NKX subfamily, which is highly conserved in all mammalian orders (Fig. 1, left and Fig. 1-1, available at https://doi.org/10.1523/JNEUROSCI.2924-18.2019. f1-1), while two conserved canonical NKX binding sites are present in nPE2 (Fig. 1, right and Fig. 1-2, available at https://doi.org/ 10.1523/JNEUROSCI.2924-18.2019.f1-2). Among all NKX family members, NKX2.1 emerged as the most likely candidate to regulate hypothalamic Pomc expression because it is present in the early developing ventral hypothalamus portion that gives rise to the arcuate nucleus (Kimura et al., 1996; Marín et al., 2002; Yee et al., 2009; Orquera et al., 2016) and continues to be expressed in this nucleus during postnatal life (Lee et al., 2001). A necessary condition to support NKX2.1 as a transactivator of hypothalamic Pomc is that both genes coexpress within the same neurons. We followed the developmental pattern of $N k \times 2.1$ expression in the ventromedial hypothalamus in sagittal sections of Pomc-EGFP mice, a validated transgenic line that expresses EGFP in most POMC neurons along the entire spatiotemporal domain, which is useful to maximize the identification of POMC neurons (Cowley et al., 2001; Nasif et al., 2015). During the early stages of hypothalamic development NKX2.1 establishes the alar-basal border that separates the suprachiasmatic nucleus ( $\mathrm{SCH}$ ) from the anterobasal medial nucleus (ABasM), and it is in this latter nucleus where POMC neurons originate (Fig. $2 d-f$; Orquera et al., 2016). The expression territory of $N k \times 2.1$ is much broader than that of Pomc extending ventrally beyond the limits of the future arcuate nucleus along the entire terminal ventromedial hypothalamus (THyVM; Fig. 2d). At the onset of Pomc expression in the mouse hypothalamus (E10.5; Japón et al., 1994), incipient Pomc-EGFP neurons are located within the dorsal limit of NKX2.1 domain (Fig. $2 a-c$ ). At E12.5, during the peak of neurogenesis of POMC neurons, all Pomc-EGFP neurons are established in the mantle zone of the developing ABasM within NKX2.1 territory and coexpress NKX2.1 (Fig. 2d-f). In the adult arcuate nucleus, Pomc-EGFP ${ }^{+}$neurons also coexpress NKX2.1 (Fig. $2 g-i$ ), in agreement with a previous report (Yee et al., 2009). Together, these results show that POMC neurons express $N k \times 2.1$ in the ventromedial hypothalamus during development and adulthood. 


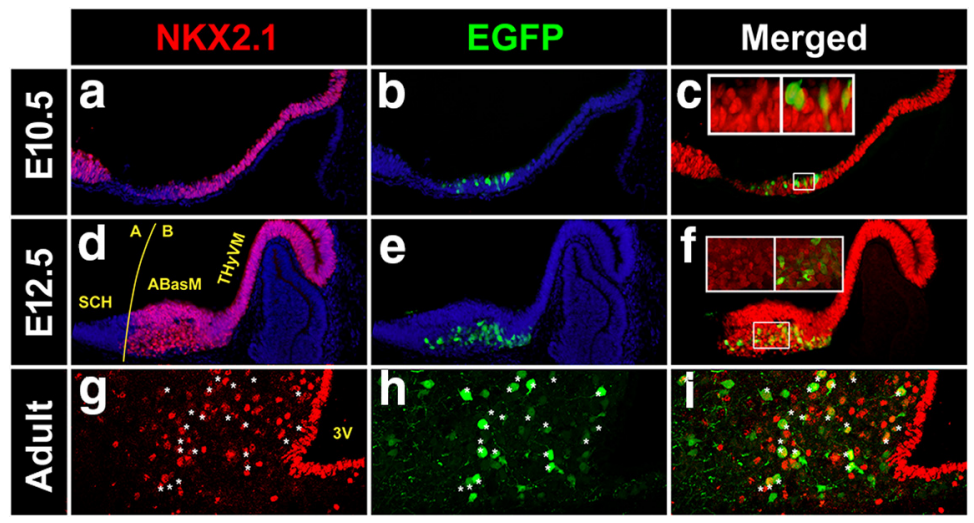

Figure 2. Pomc coexpresses with Nkx2.1 in the developing and adult mouse hypothalamus. $\boldsymbol{a}-\boldsymbol{i}$, Expression analysis of $N k x 2.1$ in sagittal cryosections of Pomc-EGFP E10.5 ( $\boldsymbol{a}-\boldsymbol{c})$ and E12.5 (d-f) mouse embryos and coronal sections of adult Pomc-EGFP mice at the level of the arcuate nucleus $(\boldsymbol{g}-\boldsymbol{i})$. Insets are magnified views showing that all EGFP ${ }^{+}$cells express Nkx2.1. A, Alar; B, basal; 3V, third ventricle. Asterisks denote examples of neurons coexpressing NKX2.1 and EGFP.
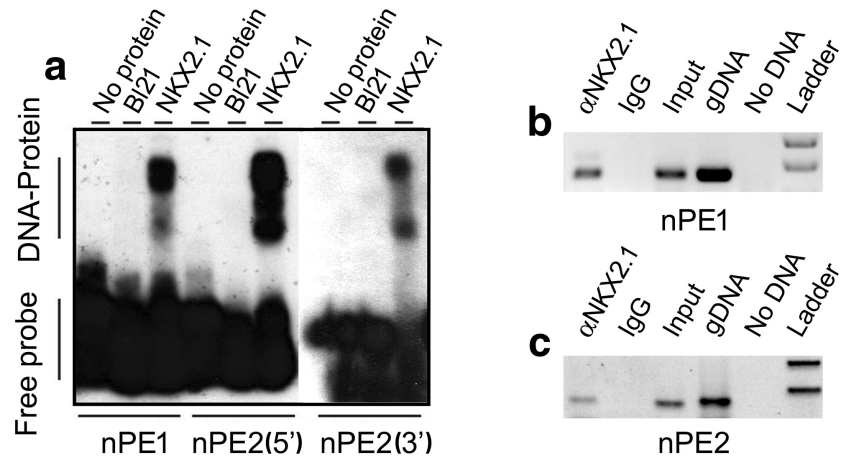

Figure 3. NKX2.1 binds to $\mathrm{nPE} 1$ and $\mathrm{nPE} 2$ sequences in vitro and in vivo. $\boldsymbol{a}, \mathrm{EMSA}$ shows that NK2.1 binds to the NKX canonical sequence present in $\mathrm{nPE} 1$ (left) and to two NKX motifs present in $\mathrm{nPE2}$ (5' site, middle; $3^{\prime}$ site, right). $\boldsymbol{b}, \boldsymbol{c}$, ChIP assays with adult mouse hypothalamus chromatin and an anti-NKX2.1 antibody that immunoprecipitates nPE1 (b) and nPE2 (c) sequences.

NKX2.1 specifically binds in vitro and in vivo to DNA elements present in nPE1 and nPE2

We then examined the in vitro binding properties of NKX2.1 in EMSAs using a bacterially expressed mouse $N k \times 2.1$ clone. We found that $30 \mathrm{bp}$ DNA probes encompassing canonical NKX motifs present in nPE1 or nPE2 were shifted when incubated with NKX2.1 bacterial extracts (Fig. 3a). To test whether NKX2.1 interacts with nPE sequences in vivo we performed a chromatin immunoprecipitation (ChIP) assay using chromatin harvested from adult mouse hypothalami. An anti-NKX2.1 antibody pulled down nPE1 and nPE2 sequences that were amplified by PCR, whereas a control IgG antibody failed to immunoprecipitate either $\mathrm{nPE}$ sequence (Fig. $3 b, c$ ).

Functional analysis of NKX binding sites present in the neuronal Pomc enhancers nPE1 and nPE2

In previous studies, we demonstrated that the sole presence of nPE1 or nPE2 in transgenic constructs is sufficient to drive reporter gene expression to arcuate POMC neurons (de Souza et al., 2005, 2011; Franchini et al., 2011). To determine the functional relevance of the two NKX binding motifs present in nPE2 (Fig. 1b), we tested whether a mutant version carrying transition mutations in these two binding sites is able to drive EGFP expression to the arcuate nucleus of transgenic mice (Fig. 4a). Both transgenes carried the mouse Pomc proximal promoter known to drive reporter gene expression exclusively to POMC pituitary cells, which allows us to discard transgenic mice carrying silent integrations (Young et al., 1998; Santangelo et al., 2007). As expected from previous studies (Franchini et al., 2011), we found that transgene nPE2Pomc-EGFP drove expression to arcuate hypothalamic neurons in three different pedigrees (Fig. $4 b)$. In contrast, four independent transgenic lines carrying the mutant version nPE2 $\left(\mathrm{NKX}^{\star}\right)$ Pomc-EGFP displayed either very few (two lines; Fig. $4 c$ ) or a complete absence of $\mathrm{EGFP}^{+}$arcuate neurons (two lines; Fig. 4d). All seven transgenic lines analyzed displayed consistent EGFP expression in the pituitary gland (Fig. $4 e-g$ ). These results indicate that the NKX binding motifs in $\mathrm{nPE} 2$ are critical for proper enhancer activity in arcuate POMC neurons.

To test the importance of the unique NKX binding site present in nPE1, we took a different genetic approach using CRISPR/ Cas9 technology to selectively delete the motif from nPE1 by targeted mutagenesis of Pomc alleles already lacking nPE2 (Lam et al., 2015). We microinjected Pomc ${ }^{+/ \triangle n P E 2}$ zygotes with an sgRNA directed to the unique NKX site present in nPE1 and obtained $\mathrm{nPE} 1(\triangle \mathrm{NKX})$ mutant alleles that, in addition, lack nPE2 $\left(\right.$ Pomc $^{n P E 1(\triangle N K X) . \Delta n P E 2}$; Fig. 4h). Pomc ${ }^{\triangle n P E 2 / \triangle n P E 2}$ E12.5 embryos of either sex showed reduced Pomc expression levels compared with $\mathrm{Pomc}^{+/+}$controls, in agreement with our previous findings (Fig. 4i,j; Lam et al., 2015). Interestingly, homozygous carriers of POMc ${ }^{n P E 1(\triangle N K X) . \triangle n P E 2}$ alleles showed even greater reductions in Pomc expression levels in the developing arcuate nucleus compared with Pomc $\triangle n P E 2 / \triangle n P E 2$ mutants, with only occasional $\mathrm{ACTH}^{+}$neurons observed (Fig. $4 i-k$ ). A quantitative analysis performed by quantitative real-time PCR (qRT-PCR) revealed that homozygous Pomc ${ }^{\triangle n P E 2 / \triangle n P E 2}$ E11.5 embryos express $27.1 \%$ of wild-type Pomc mRNA levels (Fig. $4 l$ ), similar to what has been previously reported (Lam et al., 2015). Interestingly, ablation of the NKX binding motif present in nPE1 $\left(P_{\left.0 m c^{n P E 1(\triangle N K X) . \triangle n P E 2}\right)}\right.$ further reduced Pomc expression to only $4.6 \%$ of normal levels (Fig. $4 l$ ) present in wild-type E11.5 siblings [one-way ANOVA, $F_{(28)}=48.36 ; p<0.0001$ followed by Tukey post hoc test: $\operatorname{Pomc}^{+/+}(n=5)$ vs Pomc ${ }^{\Delta n P E 2 / \Delta n P E 2}(n=8): z$ $=-4.725 ; p<0.001, P_{P o m c}^{+/+}$vs Pomc $c^{n P E 1(\Delta N K X) . \Delta n P E 2 / n P E 1}$ $(\triangle \mathrm{NKX}) . \triangle n P E 2(n=7): z=-6.302 ; p<0.001 ; P^{2} c^{\Delta n P E 2 / \Delta n P E 2}$ vs

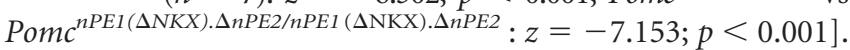
Different from Pomc $c^{\triangle n P E 2 / \triangle n P E 2}$ adult mice, which express $80 \%$ of hypothalamic Pomc mRNA levels and display normal body weight (Lam et al., 2015), homozygous male carriers of POmc $^{n P E 1(\triangle N K X) . \triangle n P E 2}$ express only $51 \%$ of Pomc mRNA levels (Fig. 4m; two-tailed Student's $t$ test: $t=4.976$; $\mathrm{df}=9, p=0.0008$ ) and are significantly heavier $(34.9 \pm 0.4 \mathrm{~g} ; n=4)$ than their wild-type littermates $(30.6 \pm 0.5 \mathrm{~g} ; n=7$; Fig. $4 n$; two tailed Student's $t$ test: $t=6.082 ; \mathrm{df}=8, p=0.0003)$. These results reveal that the NKX motif present in nPE1 is a functionally critical component of this enhancer. Altogether, our transgenic and mutant studies demonstrate that the neuronal Pomc enhancers nPE1 and nPE2 depend on their NKX binding sites to fully drive endogenous or reporter gene expression in hypothalamic neurons and suggest that NKX2.1 is critically involved in neuronal Pomc expression. 
a
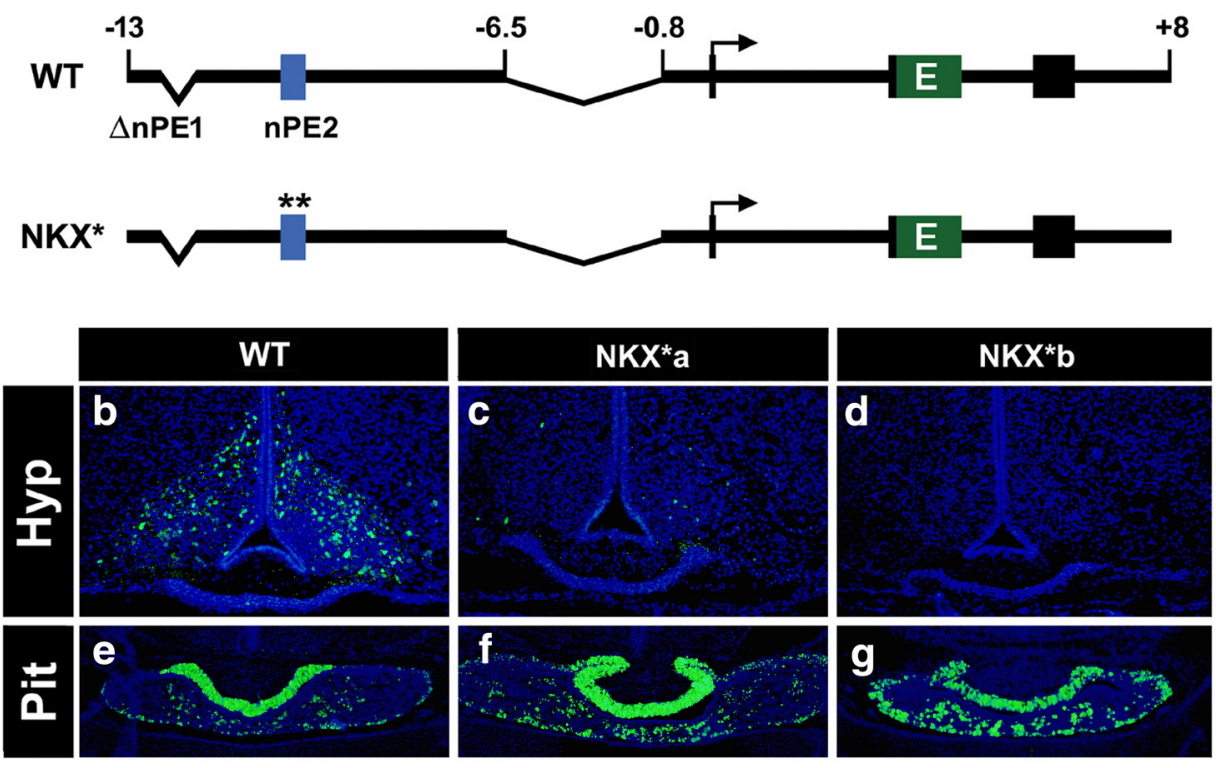

h
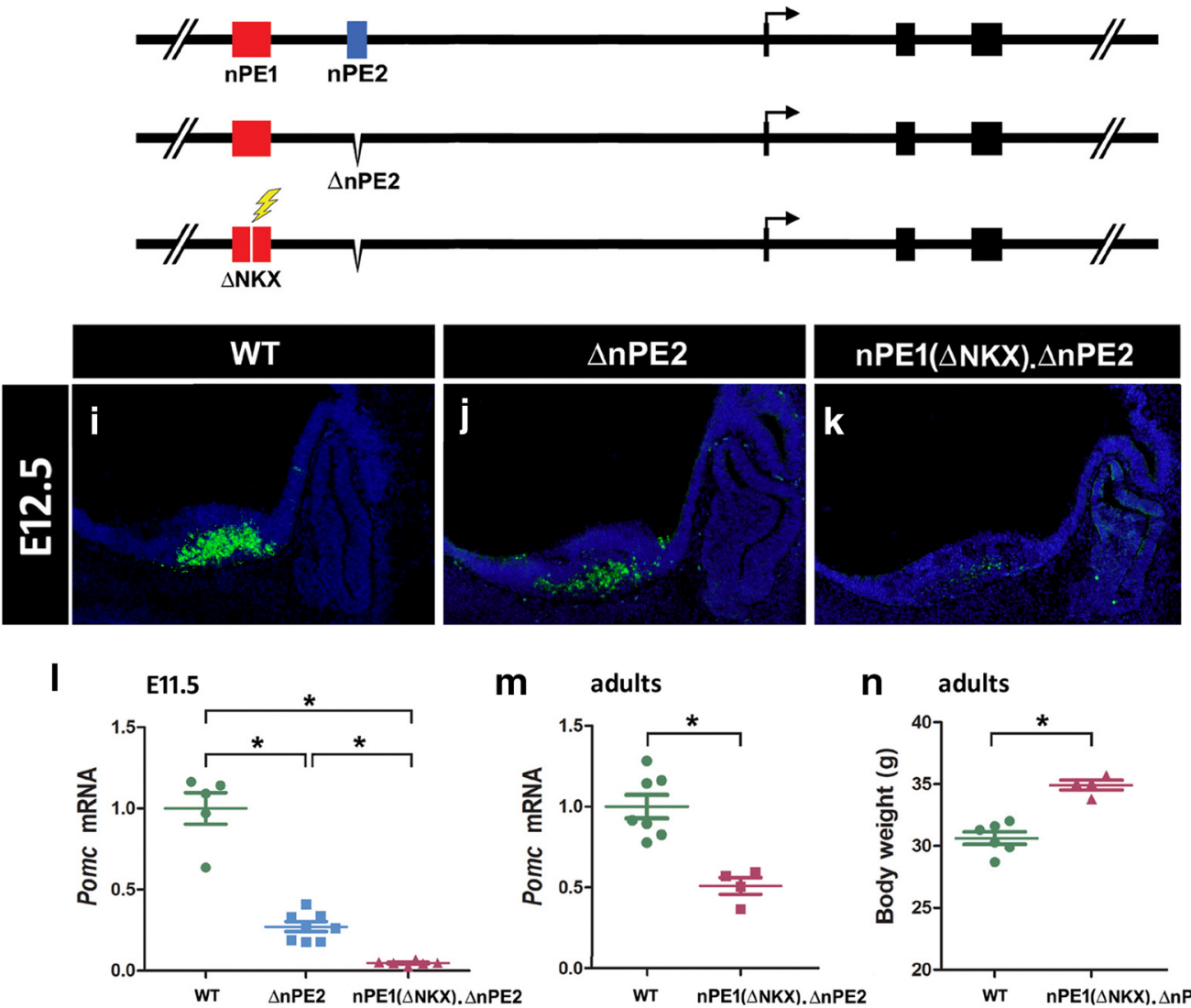

adults

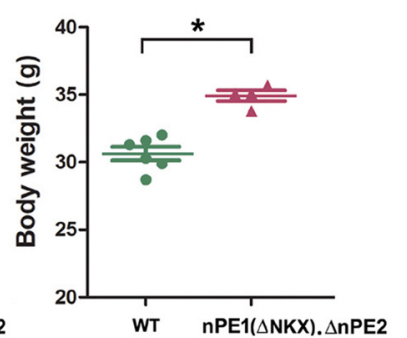

Figure 4. NKX2.1 binding sites in nPE1 and nPE2 are important for enhancer functions. $\boldsymbol{a}$, Schematic of two nearly identical transgenes constructed to study the importance of the NKX binding

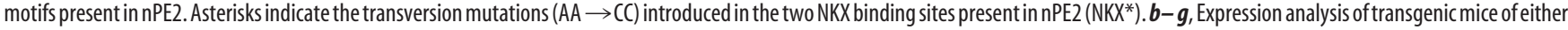
sex at PO carrying wild-type nPE2 (WT) or the mutant nPE2 version driving EGFP on coronal sections at the level of the arcuate nucleus $(\boldsymbol{b}-\boldsymbol{d})$ and the pituitary $(\boldsymbol{e}-\boldsymbol{g})$. Two independent transgenic lines carrying $n$ PE2 (NKX*) are shown $(\boldsymbol{c}, \boldsymbol{d}, \boldsymbol{f}, \boldsymbol{g}) . \boldsymbol{h}$, Schematic of the three Pom alleles analyzed at E12.5. WT mice carry intact nPE1 and nPE2, $\Delta \mathrm{nPE2}$ mice lacknPE2, and nPE1 $(\Delta \mathrm{NKX}) . \Delta \mathrm{nPE2}$ mice also carry a deletion of the NKX binding motif in nPE1 introduced by CRISPR/Cas9, indicated with a lightning symbol. $\boldsymbol{i}-\boldsymbol{k}$, Immunofluorescence analysis of Pomc expression using an anti-ACTH antibody on sagittal sections in E12.5 embryos. I, RT-qPCR analysis of Pome mRNA levels collected from Pomc ${ }^{+/+}{ }^{,}$Pomc ${ }^{\triangle A P E 2 / \triangle A P E 2}$, and Pomc ${ }^{n P E 1(\triangle N K X) . \triangle n P E 2 / n P E 1(\triangle N K X) . \triangle n P E 2}$ E11.5 embryo heads. $\boldsymbol{m}, \mathrm{RT}-\mathrm{qPCR}$ analysis of hypothalamic Pomc mRNA levels from 12-week-old Pomc ${ }^{+/+}$and Pom $\mathrm{CPE1}^{n N K X) . \triangle n P E 2 / n P E 1(\Delta N K X) . \Delta n P E 2}$ male siblings. $\boldsymbol{n}$, Body weight of same mice as in $\boldsymbol{m}$. Bars represent the mean \pm SEM. ${ }^{*} p<0.001$ 


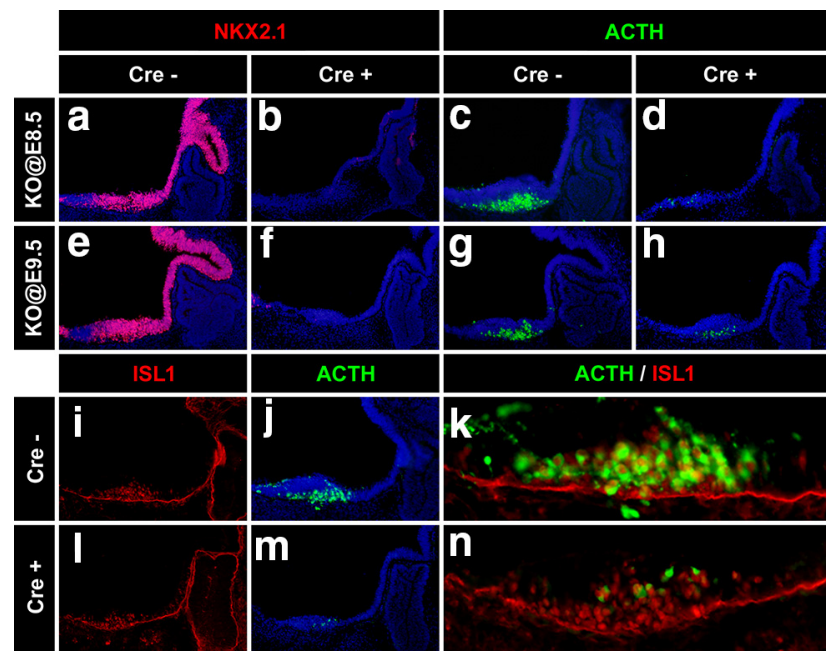

Figure 5. Hypothalamic Pomc expression is impaired in Nkx2.1 conditional knock-out mice. $\boldsymbol{a}-\boldsymbol{h}$, Immunofluorescence analysis using anti-NKX2.1 (red) and anti-ACTH (green) antibodies in sagittal cryosections of E12.5 mouse embryos injected with tamoxifen at E8.5 ( $\boldsymbol{a}-\boldsymbol{d})$ or E9.5 $(\boldsymbol{e}-\boldsymbol{h}) . \mathrm{Cre}^{+}$and $\mathrm{Cre}^{-}$embryos are littermates. $\boldsymbol{i}-\boldsymbol{n}$, Immunofluorescence analysis of E12.5 mouse embryos injected with tamoxifen at E9.5 using antibodies against ISL1 $(\boldsymbol{i}, \boldsymbol{l})$ or ACTH $(\boldsymbol{j}$, $\boldsymbol{m})$; closed-up merged pictures are also shown $(\boldsymbol{k}, \boldsymbol{n})$.

NKX2.1 is critical for the early establishment of hypothalamic melanocortin neuron identity

To investigate whether NKX2.1 participates in the establishment of the hypothalamic POMC lineage and/or in the developmental expression of Pomc, we used a conditional mutant mouse strain that allowed $N k \times 2.1$ ablation at different developmental time points. In $N k x 2.1^{\text {loxP/loxP }}$ mice, the homeodomain-encoding exon 2 is flanked by two loxP sites so that null alleles are generated upon Cre recombinase activation (Kusakabe et al., 2006). By crossing $N k \times 2.1^{\text {loxP/loxP }}$ conditional mutants with mice harboring a transgene that ubiquitously expresses a tamoxifen-inducible Cre recombinase (CAAG-CreERT; Hayashi and McMahon, 2002), we obtained inducible $N k \times 2.1^{\text {loxP/loxP }}$.CAAG-CreER mice, which, for simplicity, we named Ind $N k \times 2.1 \mathrm{KO}$. Pregnant $N k x 2.1^{\text {loxP/loxP }}$ dams mated with Ind $N k x 2.1 \mathrm{KO}$ males received a single TAM injection at different developmental time points (E8.5 and E9.5), and Ind Nkx2.1 KO and Nkx2.1 $1^{\text {loxP/loxP }}$ littermates of either sex were collected at E12.5, with the latter being used as controls. Ind $N k \times 2.1 \mathrm{KO}$ embryos receiving TAM at E8.5 (Nkx2.1KO@E8.5) showed a complete absence of NKX2.1 when evaluated in E12.5 sagittal sections (Fig. $5 a, b$ ), demonstrating that this dose of TAM induced efficient recombination of the conditional $N k x 2.1^{l o x P}$ alleles. Nkx2.1KO@E8.5 embryos showed a thinning of the ventral neuroepithelium at the level of the future hypothalamus and lack of infundibulum, as found in $N k \times 2.1^{-/-}$ mice (Kimura et al., 1996). Immunofluorescence performed with an anti-ACTH antibody in Nkx2.1KO@E8.5 embryos showed only a few POMC cells, in contrast to what was observed in control embryos lacking CreER (Fig. 5c,d). TAM injected at E9.5 also induced inactivation of the $N k \times 2.1$ conditional alleles as evidenced by the complete lack of NKX2.1 signal in Nkx2.1KO@E9.5 embryos that showed an overall normal morphology (Fig. 5e,f). In the absence of NKX2.1, the number of POMC ${ }^{+}$cells was markedly reduced in Nkx2.1KO@E9.5 embryos (Fig. 5g,h; control: $58.25 \pm 7.05$ cells/slice, $n=4$; Nkx2.1KO@E9.5: $25.25 \pm 0.25$ cells/slice, $n=4$. GLM Poisson distribution, $z=-7.02, p<0.0001)$. These results indicate that $N k \times 2.1$ ablation from early stages of embryonic development impairs the onset of Pomc expression. Thus, NKX2.1 is nec-

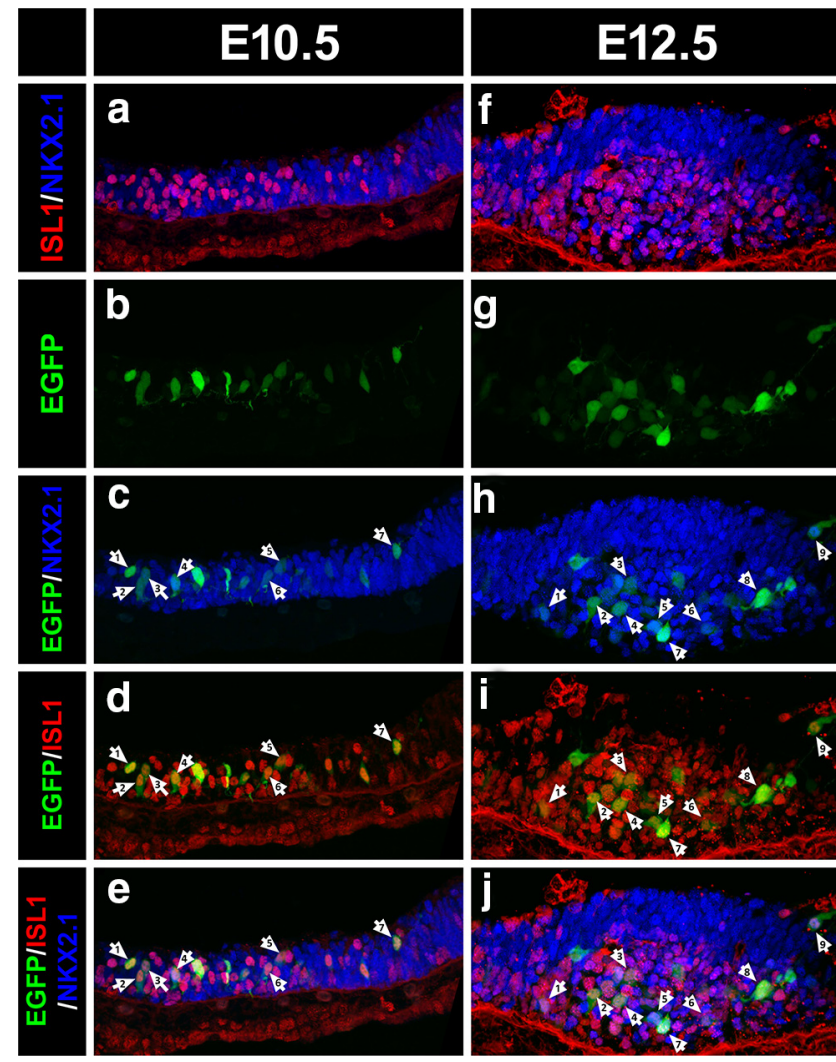

Figure 6. Overlapping expression patterns of Nkx2.1, Is/1, and Pomc in the developing hy pothalamus. $\boldsymbol{a}-\boldsymbol{j}$, Immunofluorescence analysis using anti-NKX2.1 (blue) and anti-ISL1 (red) antibodies in sagittal cryosections of E10.5 $(\boldsymbol{a}-\boldsymbol{e})$ and E12.5 (f-j) Pomc-EGFP mouse embryos. Confocal images showing superimposed NKX2.1- and ISL1-immunopositive neurons $(\boldsymbol{a}, \boldsymbol{f})$ and triple merged with Pomc-EGFP-immunoreactive neurons $(\boldsymbol{e}, \boldsymbol{j})$. Arrows denote examples of neurons coexpressing NKX2.1, ISL1, and EGFP.

essary to fully activate hypothalamic Pomc expression and, consequently, to establish the entire set of melanocortin neurons.

In a previous study (Nasif et al., 2015), we showed that the onset of hypothalamic Pomc expression at E10.5 depends on the earlier expression of the TF ISL1, which starts at E10.0 in maturing postmitotic neurons of the future arcuate nucleus. Given that the onset of $N k \times 2.1$ expression in the developing hypothalamus precedes that of Isl1, we tested the hypothesis that NKX2.1 activates Pomc expression via ISL1, alternatively to its direct effect acting on the NKX binding sites present in nPE1 and nPE2. We found, however, normal ISL1 immunoreactivity in ventromedial hypothalamic neurons of Nkx2.1KO@E9.5 embryos (Fig. 5i,l), indicating that Isl1 does not seem to be downstream of $N k x 2.1$. Furthermore, in the absence of NKX2.1, ISL1 was unable to promote normal Pomc expression, as evidenced by the large number of ISL1-immunoreactive neurons that do not express Pomc in the hypothalamus of Nkx2.1KO@E9.5 embryos (Fig. 5l-n), compared with what was found in their $N k x 2.1^{\text {loxP/loxP }}$ siblings (Fig. $5 i-k)$. These results, together with those shown in the sections above, support the idea that NKX2.1 activates Pomc expression directly.

\section{Nkx2.1 expression in ISL1 neurons is critical for} hypothalamic Pomc expression

To limit Nkx2.1 ablation to the lineage leading to arcuate POMC neurons, we decided to inactivate $N k \times 2.1$ alleles specifically in neurons expressing Isl1. We found that the pattern of Isll expression within the presumptive arcuate nucleus at E10.5 overlaps 
with that of $N k x 2.1$ (Fig. $6 a$ ), and that Pomc-EGFP ${ }^{+}$cells (Fig. 6b) coexpress Isl1 and $N k \times 2.1$ (Fig. $6 c-e$ ). A similar pattern of triple coexpression was observed in E12.5 embryos (Fig. 6f-j).

By mating $N k \times 2.1^{\text {loxP/loxP }}$ mice with a knock-in strain that expresses Cre under the transcriptional control of Isl1 (Yang et al., 2006), we obtained $N k x 2.1^{\text {loxP/loxP }}$. Isl1 ${ }^{+/ \text {cre }}$ mice, which we named Isl1 Nkx2.1KO. Isl1 Nkx2.1KO embryos at E11.5 showed only $27.5 \pm 5.8 \%$ of hypothalamic POMC neurons compared with Nkx2.1 $1^{\text {loxP/loxP }}$ controls (Fig. $7 a, b$; twotailed Student's $t$ test: $t=11.62, \mathrm{df}=5$, $p<0.0001$; control, $n=4$; Isl1 Nkx2.1KO, $n=3)$. In these embryos, the hypothalamic pattern of $N k \times 2.1$ expression (Fig. $7 c, d)$ is only altered in the mantle zone of the developing arcuate nucleus, where most ISL1 ${ }^{+}$cells reside (Fig. $7 e-h$ ). As observed in Nkx2.1KO@E9.5 embryos (Fig. 5i,l), Isl1 Nkx2.1KO showed normal ISL1 levels (Fig. 7e,f). Expression analysis of the proneuronal markers ASCL1 (Fig. $7 i, j$ ) and Neurogenin-3 (Fig. $7 k, l$ ) in the developing hypothalamus showed no differences between control and Isl1 Nkx2.1KO E11.5 embryos, confirming that the selective ablation of $N k x 2.1$ from ISL1 postmitotic neurons does not affect neurogenesis in this brain region. Analysis of Isl $1 N k \times 2.1 \mathrm{KO}$ embryos at E12.5 also showed a great reduction of $\mathrm{ACTH}^{+}$neurons (Fig. $7 m, n$ ) due to the loss of NKX2.1 in this region (Fig. 7o,p). Thus, the lack of $N k \times 2.1$ expression specifically in ISL1 ${ }^{+}$neurons impairs hypothalamic Pomc expression and confirms the critical role of NKX2.1 in the early establishment of melanocortin neuron identity.

\section{Selective ablation of $N k x 2.1$ from POMC neurons impairs Pomc} expression and increases body weight To investigate whether NKX2.1 participates in arcuate Pomc expression specifically in POMC neurons, we crossed Nkx2.1 $1^{\text {loxP/loxP }}$ mice with a BAC Pomc-Cre transgenic line ( $\mathrm{Xu}$ et al., 2005). In the resulting PomcNkx2.1 KO mice, the $N k \times 2.1$ alleles are inactivated in POMC neurons once Pomc-Cre expression begins. Immunofluorescence analysis of PomcNkx2.1KO embryos at E12.5 showed that the number and location of POMC neurons in the developing arcuate nucleus did not differ from those observed in control littermates (Fig. $8 a, b$; control: $66.5 \pm 6.84$ cells/slice, $n=4$; KO: $55.3 \pm 7.57$ cells/slice, $n=4$; GLM binomial distribution, $z$ $=-1.22, p=0.222$ ), a similar result to what we found in E15.5 embryos (Fig. $8 c$, d; control: $274 \pm 6.5$ cells/slice, $n=2$; KO:

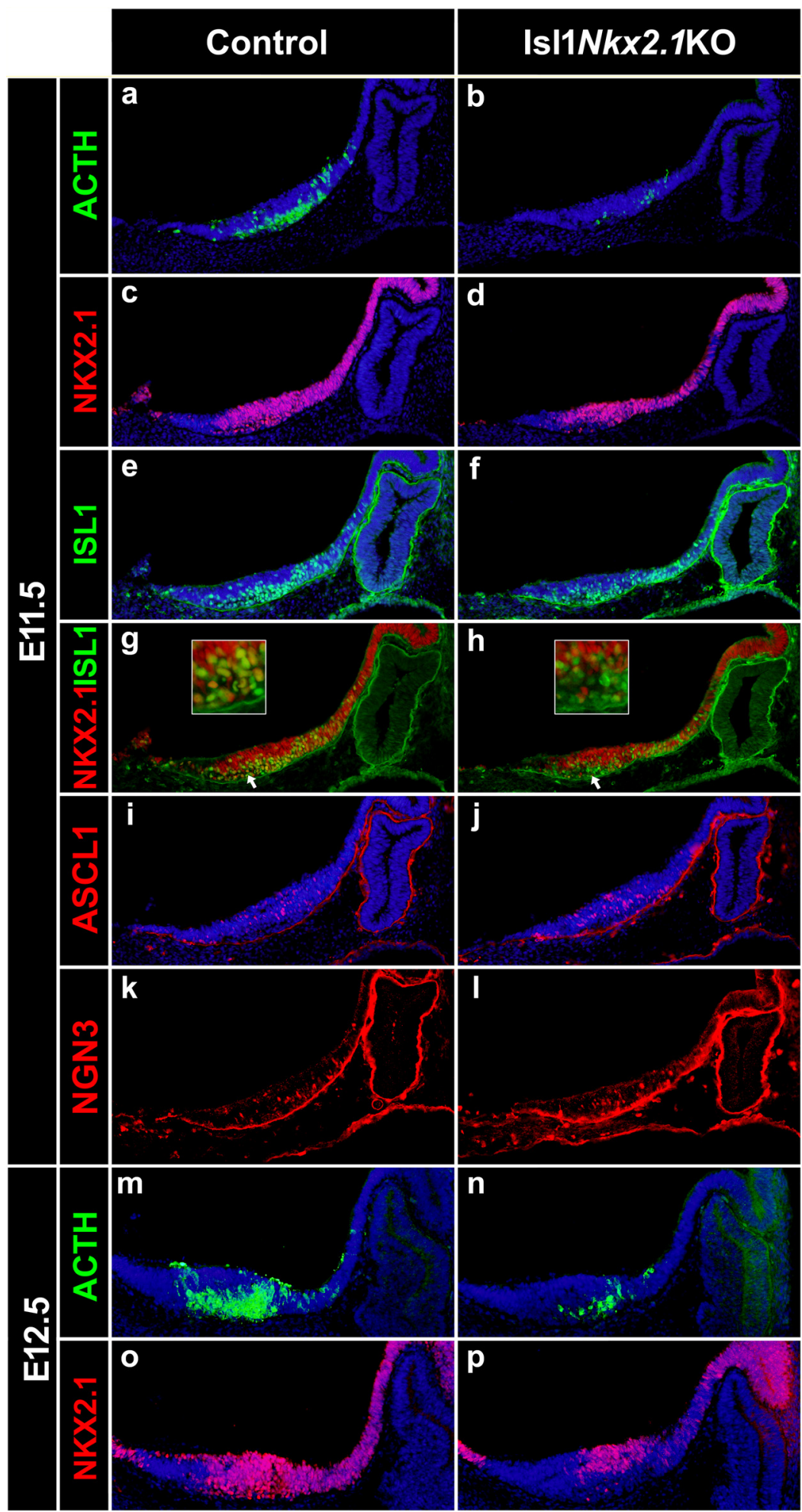

Figure 7. Specific deletion of $N k \times 2.1$ from ISL1 ${ }^{+}$neurons impairs the onset of Pomc expression. $\boldsymbol{a}-\mathbf{I}$, Immunofluorescence analysis in sagittal cryosections of E11.5 embryos using anti-ACTH $(\boldsymbol{a}, \boldsymbol{b})$, anti-NKX2.1 $(\boldsymbol{c}, \boldsymbol{d})$, anti-ISL1 $(\boldsymbol{e}, \boldsymbol{f})$, anti-ASCL1 $(\boldsymbol{i}, \boldsymbol{j})$, and anti-NGN3 $(\boldsymbol{k}, \boldsymbol{I})$ antibodies. $\boldsymbol{g}, \boldsymbol{h}$, Loss of Nkx2.1 in ISL1 ${ }^{+}$neurons is evident by double labeling. Arrows point to the mantle zone of the mediobasal hypothalamus detailed in the insets above. $\boldsymbol{m}-\boldsymbol{p}$, E12.5 Is $11 \mathrm{Nk} \times 2.1 \mathrm{~K} 0$ embryos show reduced numbers of $\mathrm{ACTH}^{+}$neurons compared with controls $(\boldsymbol{m}, \boldsymbol{n})$ and loss of NKX2.1 in the mantle zone $(\boldsymbol{o}, \boldsymbol{p})$. DAPI is shown in blue.
$258.5 \pm 11.0$ cells/slice, $n=2$; GLM binomial distribution, $z=$ $-0.95, p=0.342$ ), and in contrast to what we found when $N k \times 2.1$ was ablated at earlier time points (Figs. 5, 7). These results indicate that NKX2.1 plays a crucial role in the onset of hypothalamic 

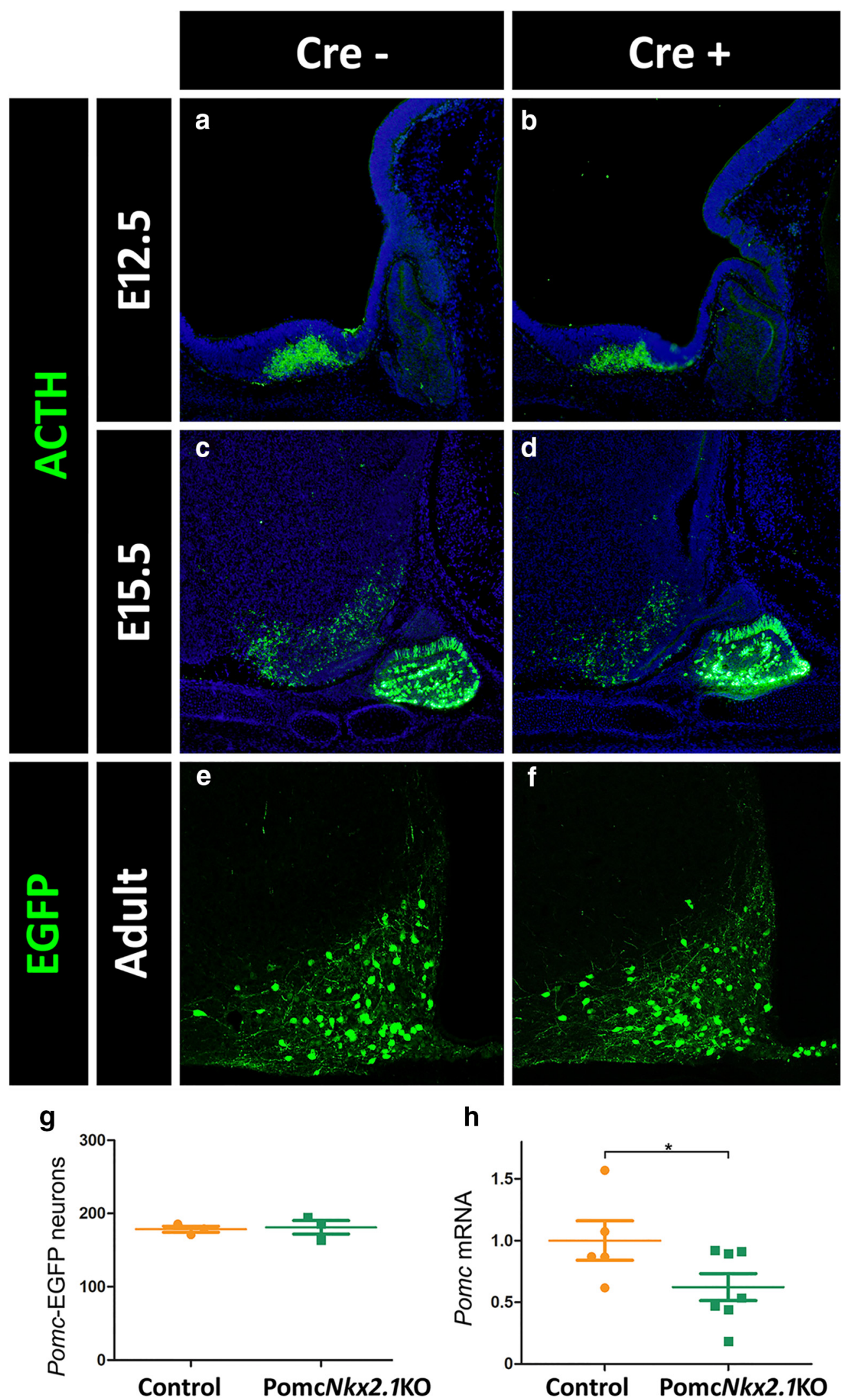

Figure 8. Nkx2.7-specific deletion in POMC neurons. $\boldsymbol{a}-\boldsymbol{d}$, The number of hypothalamic ACTH ${ }^{+}$cells in PomcNkx2.1KO mice is normal at E12.5 $(\boldsymbol{a}, \boldsymbol{b})$ and E15.5 $(\boldsymbol{c}, \boldsymbol{d})$. $\boldsymbol{e}-\boldsymbol{g}$, The number of POMC neurons in the adult arcuate nucleus of PomcNkx2.7 KO mice is normal, as assessed in Pomc-EGFP.PomcNkx2.7KO mice. $\boldsymbol{h}$, Quantitative RT-PCR shows reduced Pomc mRNA levels in the hypothalamus of adult PomcNkx2.1K0 mice. Values represent the mean \pm SEM. ${ }^{*} p<0.05$. 


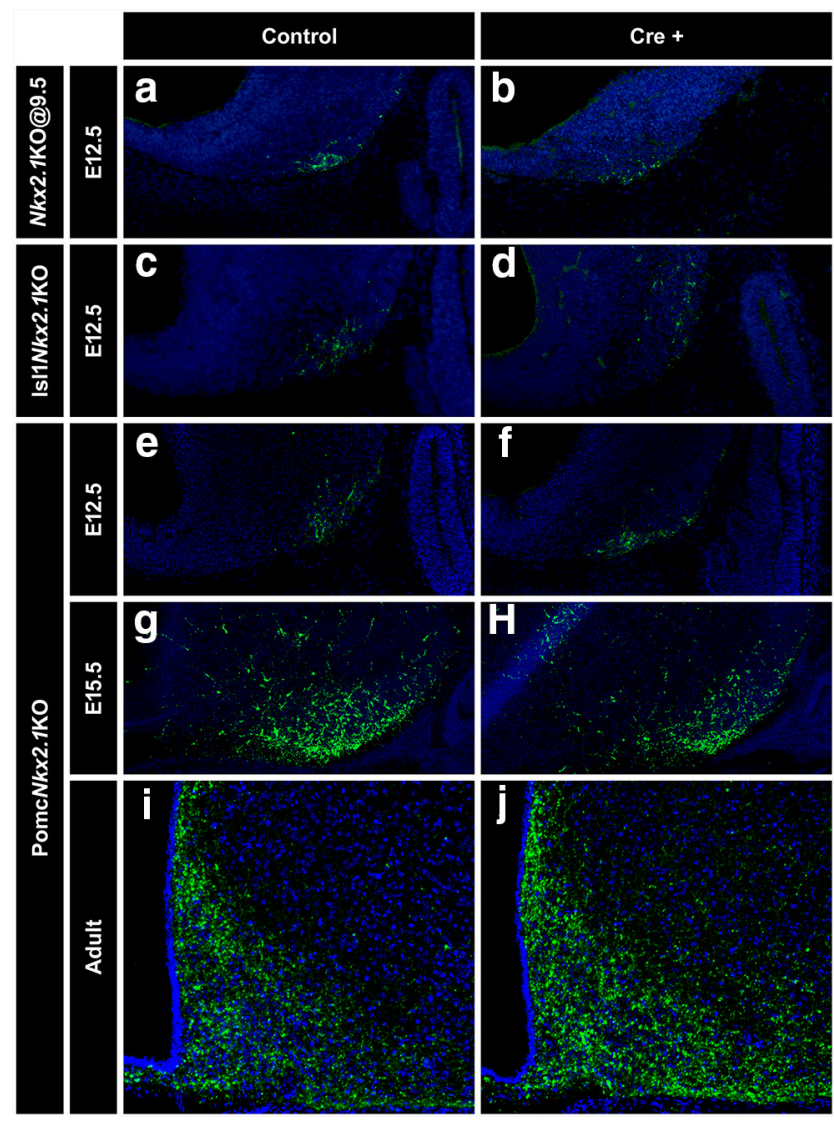

Figure 9. Hypothalamic NPY neurons in conditional Nkx2.1 mutant mice. $\boldsymbol{a}-\boldsymbol{f}$, The number of $\mathrm{NPY}^{+}$neurons is normal in Nkx2.7K0@E9.5 (b), Is11 Nkx2.7KO (d) , and PomcNkx2.7KO (f) E12.5 mouse embryos compared with their corresponding control littermates $(\boldsymbol{a}, \boldsymbol{c}, \boldsymbol{e})$, as determined by immunofluorescence using anti-NPY (green) antibody on sagittal cryosections. $\boldsymbol{g}-\boldsymbol{j}$, The signal density of NPY ${ }^{+}$cells in PomcNkx2.1K0 mice is also normal at E15.5 $(\boldsymbol{g}, \boldsymbol{h})$ and in the adult arcuate nucleus $(i, j)$.

Pomc expression and early specification of melanocortin neurons identity, and that once Pomc expression and melanocortin neurons are established, NKX2.1 function is less prominent. Given that Nkx2.1 and Pomc also coexpress in the arcuate nucleus during postnatal life, we decided to evaluate whether NKX2.1 plays a role in the transcriptional regulation of Pomc in adult mice. We found that the number of POMC neurons in the adult arcuate nucleus was normal, as assessed in Pomc-EGFP.PomcNkx2.1 KO compound mice (Fig. $8 e-g$; control: $181 \pm 9.2$ cells/slice, $n=3$; KO: $179 \pm 4.2$ cells/slice, $n=3$; GLM binomial distribution, $z=$ $0.274, p=0.784)$. However, a qRT-PCR study showed that hypothalamic Pomc mRNA levels from 20-week-old PomcNkx2.1KO male mice are $38 \%$ lower than those found in $N k x 2.1^{\text {loxP } / l o x P}$ control littermates (Fig. $8 h$; one-tailed Student's $t$ test, $t=2.022, \mathrm{df}=$ $10, p=0.0354$; control: $n=5$; KO: $n=7$ ). In agreement with the reduction in Pomc mRNA levels, 20-week-old PomcNkx2.1KO males were $11.3 \%$ heavier than their control siblings [control: $29.8 \pm 0.57 \mathrm{~g}(n=7)$; PomcNkx2.1KO: $33.0 \pm 0.86 \mathrm{~g}(n=11)$, $t=2.767, \mathrm{df}=16, p=0.014$, two-tailed Student's $t$ test $]$. In addition, the livers and retroperitoneal fat pads of PomcNkx2.1KO mice were $15 \%$ and $148 \%$ heavier than those from control littermates, respectively, whereas other fat pads showed no statistically significant difference [livers: control: $1.20 \pm 0.05 \mathrm{~g}(n=6)$; PomcNkx2.1KO: $1.38 \pm 0.05 \mathrm{~g}(n=7), t=$ 2.741, $\mathrm{df}=11, p=0.019$, two-tailed Student's $t$ test; retroperitoneal fat: control: $81.6 \pm 23.2 \mathrm{mg}(n=7)$; PomcNkx2.1KO:
$202.6 \pm 40.1 \mathrm{mg}(n=10), t=2.326, \mathrm{df}=15, p=0.0344$, two-tailed Student's $t$ test]. Overall, our results demonstrate that NKX2.1 participates in a cell-autonomous manner in attaining normal Pomc expression levels in the adult arcuate nucleus and, consequently, in the regulation of normal body weight homeostasis.

\section{The number of hypothalamic NPY neurons is not altered in} conditional $N k x 2.1$ mutant mice

POMC and NPY/AGRP arcuate neurons play antagonistic roles in the regulation of food intake. Because a population of NPY/ AGRP neurons appears to originate from earlier Pomc-expressing progenitors (Padilla et al., 2010; Sanz et al., 2015), we analyzed the number of hypothalamic NPY/AGRP neurons in the three conditional Nkx2.1 mutant mouse models described above (Figs. $5,7,8)$. Because the onset of $N p y$ expression (E12.5) in the mouse developing hypothalamus precedes that of Agrp (E15.5), we used an anti-NPY antibody to label NPY neurons at E12.5 in Nkx2.1KO@E9.5 and Isl1 Nkx2.1KO embryos, as well as in their corresponding controls. The number of NPY neurons at E12.5 was not altered after ablating $N k \times 2.1$ from the entire brain at E9.5 (Fig. 9a, $b$; control: $17.3 \pm 3.8$ cells, $n=3$; KO: $22.7 \pm 0.88$ cells, $n=3$; GLM Poisson distribution, $z=1.46, p=0.15$ ), or specifically from ISL1 ${ }^{+}$neurons (Fig. $9 c$, $d$; control: $11.7 \pm 2.3$ cells, $n=$ 3; Isl1 Nkx2.1KO: $11.6 \pm 0.51$ cells, $n=5$; GLM binomial distribution, $z=-0.027, p=0.98)$, in clear contrast to what we found for POMC neurons (Figs. $5 g, h$, Fig. $7 m, n$ ), respectively. Similarly, the number of arcuate NPY neurons was not affected in PomcNkx2.1KO E12.5 embryos [Fig. 9e,f; control: $16.3 \pm 2.6$ cells, $n=4$; KO: $15.5 \pm 1.9$ cells, $n=4$; GLM Poisson distribution, $z=-0.266, p=0.79)$, E15.5 embryos; Fig. $9 g$, $h$; control: $52.3 \pm 0.95$ densitometry arbitrary units (A.U.), $n=2$; KO: $47.4 \pm 1.3$ A.U., $n=2$; two-tailed Student's $t$ test: $t=3.104$, $\mathrm{df}=2, p=0.09$ ] or in adults (Fig. 9i,j; control: $46.8 \pm 0.99$ A.U., $n=3$; KO: $44.7 \pm 1.14$ A.U., $n=3$; two-tailed Student's $t$ test: $t=$ $1.38, \mathrm{df}=4, p=0.24)$. These results suggest that the reduction in hypothalamic Pomc expression elicited by the early ablation of $N k \times 2.1$ does not alter the number of hypothalamic NPY neurons.

\section{Discussion}

In this study, we combined molecular, genetic, cellular, and functional approaches to demonstrate that the homeodomain transcription factor NKX2.1 plays a crucial role in establishing the early identity of melanocortin neurons by promoting the onset of Pomc expression in the ventral hypothalamus. Specifically, we show the following: (1) the neuronal Pomc enhancers nPE1 and nPE2 contain canonical NKX binding sites, which are highly conserved in all mammalian orders; (2) Pomc coexpresses with $N k \times 2.1$ from its onset at E10.5 and throughout the entire life span in the arcuate nucleus; (3) NKX2.1 binds in vitro to DNA fragments from nPE1 and nPE2 carrying NKX binding motifs and in vivo to $\mathrm{nPE} 1$ and $\mathrm{nPE} 2$ in hypothalamic chromatin extracts; (4) the NKX binding motifs present in $\mathrm{nPE} 1$ and $\mathrm{nPE} 2$ are essential for their enhancer activity in arcuate neurons; (5) early expression of $N k x 2.1$ in the developing ventral hypothalamus is necessary for the onset of hypothalamic Pomc expression; (6) Nkx2.1 ablation from hypothalamic ISL1 ${ }^{+}$neurons prevents Pomc expression demonstrating that the sole presence of ISL1 is unable to activate Pomc; and (7) Nkx2.1 ablation from POMC neurons reduces 38\% Pomc mRNA levels and induces a mild increase in body weight and adiposity in adult mice.

$N k \times 2.1$ is expressed as early as E7.5 in the anterior portion of the mouse neural tube and hours later is found in neuronal pro- 
genitors of the hypothalamic anlage, where it specifies ventral lineages while repressing dorsal and alar fates (Kimura et al., 1996; Marín et al., 2002). Ablation of Nkx2.1 at this early stage impairs the formation of the ventral hypothalamic primordium, which undergoes dorsalization (Kimura et al., 1996). In addition to this essential early role defining the ventral identity of the developing hypothalamus, $N k \times 2.1$ is later expressed in postmitotic cells, suggesting that it may also be involved in the differentiation and maintenance of mature cellular phenotypes in this brain region (Sussel et al., 1999; Yee et al., 2009). In fact, we found that the early ablation of $N k \times 2.1$ induced a major deficit in the number of POMC neurons that normally arise in the ventral hypothalamus at E11.5 and extend their proliferation a day later. This impairment was particularly observed in Nkx2.1KO@E9.5 and Isl1 Nkx2.1KO embryos, two conditional mutant models in which $N k \times 2.1$ ablation precedes the onset of hypothalamic Pomc expression, but not in PomcNkx2.1KO embryos, which still express normal levels of NKX2.1 by the time Pomc starts expressing in the developing hypothalamus. This difference reveals the critical role of NKX2.1 in the early expression of hypothalamic Pomc. Thus, the multiple developmental roles that NKX2.1 plays in the future ventral hypothalamus assure first, the formation of the tuberal portion of the mediobasal hypothalamus from where the arcuate nucleus originates; second, the determination of the identity of POMC neurons in this region and; third, the maintenance of high expression levels of Pomc to regulate satiety and body weight. The role of NKX2.1 in postnatal Pomc expression is consistent with its presence in POMC neurons in the adult arcuate nucleus and with its binding in vivo to the Pomc enhancers nPE1 and nPE2 in adult mouse hypothalami.

Our results shed light on the multistep genetic program that NKX2.1 initiates in the anterior tip of the neural tube during the early developmental stages of the anterior brain, and that ends up with the integration of fully functional neuronal circuits, including those controlling food intake and energy balance. This complex program, strongly dependent on NKX2.1, includes the morphogenesis of the arcuate nucleus followed by the birth and maturation of arcuate neurons expressing Pomc. The execution of this elaborate developmental program has been recently mimicked in vitro using, at starting points, human embryonic stem cells or induced pluripotent stem cells (Merkle et al., 2015; Wang et al., 2015). Upon treatment with the morphogen Sonic Hedgehog Homolog and inhibitors of the SMAD and Notch pathways, these cells acquire features of ventral hypothalamic neural progenitors including the expression of Nkx2.1 (Wang et al., 2015). Further inhibition of Notch signaling differentiates NKX2.1 ${ }^{+}$ cells to a population of neuronal phenotypes typical of the arcuate nucleus including POMC, NPY/AGRP, somatostatin, and TH/dopamine (Wang et al., 2015).

In addition to its critical role in the developmental plan of the ventral hypothalamus and telencephalic medial ganglionic eminence (Marín et al., 2002), NKX2.1 drives the differentiation of particular cell types in peripheral organs such as the thyroid gland (Kusakabe et al., 2006) and the lungs (Kimura et al., 1996). As we found in hypothalamic POMC neurons, NKX2.1 participates not only in the morphogenesis of the thyroid gland but also in the differentiation of thyrocytes and in the regulation of the expression of thyroid-specific genes such as thyroglobulin (Miccadei et al., 2002). The concomitant function of NKX2.1 in these three organs is evident in a familial clinical condition known as brainlung-thyroid syndrome, characterized by congenital hypothyroidism, infant respiratory distress, and benign hereditary chorea, which is found in patients carrying heterozygous mis- sense or nonsense mutations in the coding region of human NKX2.1 (Miccadei et al., 2002). Some NKX2.1 mutations involve two or just one of these conditions, and, in addition, familial cases of ataxia and pituitary abnormalities have been reported (Veneziano et al., 2014). Although none of these cases reported comorbidity with overweight or obesity, our observation that $N k \times 2.1$ deficiency in POMC neurons induces increased adiposity and overweight in the mouse may be of biomedical relevance since polymorphisms leading to very low levels of NKX2.1 in the ventral hypothalamus or in NKX binding motifs in neuronal POMC enhancers may reduce $P O M C$ expression and consequently impair the control of food intake and energy balance. Innumerable genome-wide association studies have been performed during the last decades identifying $>100$ different loci potentially associated with high body mass index, type 2 diabetes, increased adiposity or high leptin levels (Thorleifsson et al., 2009; Wheeler et al., 2013; Gaulton et al., 2015). Although the individual contribution of the vast majority of these variants is quantitatively irrelevant, a polymorphic sequence present in a conserved intronic region of the FTO gene gained special significance because its statistical power has been replicated in other unrelated studies (Scuteri et al., 2007; Loos and Bouchard, 2008). Further genetic studies showed that this conserved intronic sequence is a tissuespecific enhancer that controls not FTO but a distant gene coding for the TF IRX3 (Smemo et al., 2014). Another exceptional single locus is $P O M C$, since a number of genome-wide studies have found highly significant linkage scores between obesity-related traits and a genomic segment in chromosome 2 near POMC (Comuzzie et al., 1997; Rotimi et al., 1999; Delplanque et al., 2000). Although polymorphisms in POMC coding sequences do not appear to account for these associations (Hixson et al., 1999), it is likely that mutations in noncoding regulatory elements may alter $P O M C$ transcript levels and modify the relative amount of central melanocortins.

Our finding that NKX2.1 binds to canonical NKX binding motifs present in nPE1 and nPE2 adds to the adaptive partial redundancy of arcuate Pomc expression that relies on the presence of these two enhancers for full transcriptional activity (Lam et al., 2015). During mammalian evolution, nPE1 and nPE2 were independently exapted (co-opted) from different types of retroposons (Franchini et al., 2011). This long-lasting evolutionary process in which two different retroposon-derived sequences became functional neuronal Pomc enhancers involved the independent acquisition of NKX binding sites in each of them, as we previously found for ISL1 binding sites (Nasif et al., 2015). Because the individual ablation of Nkx2.1 or Isl1 from early developmental stages prevents the onset of hypothalamic Pomc expression, we conclude that the combinatorial presence of NKX2.1 and ISL1 is necessary to determine the identity of arcuate melanocortin neurons. In fact, in this study we show that Pomc is expressed in NKX2.1 ${ }^{+}, \mathrm{ISL}_{1}{ }^{+}$neurons. However, since the number of neurons coexpressing these two TFs in the arcuate nucleus greatly exceeds that of Pomc, it is clear that NKX2.1 and ISL1 are not sufficient for arcuate-specific Pomc expression and that at least another TF, yet to be discovered, is necessary to dictate the identity of POMC neurons. This functionally redundant ensemble of enhancers (nPE1 and nPE2) and TFs (ISL1 and NKX2.1) orchestrate neuron-specific Pomc expression at relatively high levels in such a cooperative way that the assessment of the relative contribution of each component by individual mutations is difficult to establish.

The relationship between hypothalamic Pomc mRNA levels and body weight is not linear. After studying a variety of hypo- 
morphic mutant mice, we have come to the conclusion that only after arcuate Pomc mRNA levels drop to $60-50 \%$ of normal values do mild and late-onset overweight develop. From 50\% to $20 \%$ of Pomc mRNA levels, overweight and adiposity slightly increase, and only when Pomc levels are lower than 10\% earlyonset extreme obesity is apparent (Lam et al., 2015; Nasif et al., 2015). In the extreme case of total absence of neuronal Pomc expression hyperphagia amd adiposity are maximal (Bumaschny et al., 2012). Hypothalamic Pomc mRNA levels in PomcNkx2.1KO adult males were shown to be $62 \%$, and these mice are mildly overweight (11.3\%), whereas homozygous nPE1( $\Delta$ NKX). $\Delta$ nPE2 mice expressing 51\% Pomc are 14\% heavier than controls, indicating that the specific ablation of the TF NKX2.1 in POMC neurons or the targeted deletion of their cognate binding sites may result in similar deficits of Pomc expression. Altogether, we conclude that NKX2.1 and the NKX binding sites of the neuronal enhancers of Pomc participate in a multicomponent system that assures normal levels of hypothalamic Pomc expression and body weight regulation.

\section{References}

Aponte Y, Atasoy D, Sternson SM (2011) AGRP neurons are sufficient to orchestrate feeding behavior rapidly and without training. Nat Neurosci 14:351-355.

Benoit SC, Air EL, Coolen LM, Strauss R, Jackman A, Clegg DJ, Seeley RJ, Woods SC (2002) The catabolic action of insulin in the brain is mediated by melanocortins. J Neurosci 22:9048-9052.

Bumaschny VF, Yamashita M, Casas-Cordero R, Otero-Corchón V, de Souza FS, Rubinstein M, Low MJ (2012) Obesity-programmed mice are rescued by early genetic intervention. J Clin Invest 122:4203-4212.

Chen Y, Lin YC, Kuo TW, Knight ZA (2015) Sensory detection of food rapidly modulates arcuate feeding circuits. Cell 160:829-841.

Chhabra KH, Adams JM, Jones GL, Yamashita M, Schlapschy M, Skerra A, Rubinstein M, Low MJ (2016) Reprogramming the body weight set point by a reciprocal interaction of hypothalamic leptin sensitivity and pomc gene expression reverts extreme obesity. Mol Metab 5:869-881.

Cho HH, Cargnin F, Kim Y, Lee B, Kwon RJ, Nam H, Shen R, Barnes AP, Lee JW, Lee S, Lee SK (2014) Isll directly controls a cholinergic neuronal identity in the developing forebrain and spinal cord by forming cell typespecific complexes. PLoS Genet 10:e1004280.

Comuzzie AG, Hixson JE, Almasy L, Mitchell BD, Mahaney MC, Dyer TD, Stern MP, MacCluer JW, Blangero J (1997) A major quantitative trait locus determining serum leptin levels and fat mass is located on human chromosome 2. Nat Genet 15:273-276.

Cowley MA, Smart JL, Rubinstein M, Cerdán MG, Diano S, Horvath TL, Cone RD, Low MJ (2001) Leptin activates anorexigenic POMC neurons through a neural network in the arcuate nucleus. Nature 411:480-484.

Delplanque J, Barat-Houari M, Dina C, Gallina P, Clément K, Guy-Grand B, Vasseur F, Boutin P, Froguel P (2000) Linkage and association studies between the proopiomelanocortin (POMC) gene and obesity in caucasian families. Diabetologia 43:1554-1557.

de Souza FS, Santangelo AM, Bumaschny V, Avale ME, Smart JL, Low MJ, Rubinstein M (2005) Identification of neuronal enhancers of the proopiomelanocortin gene by transgenic mouse analysis and phylogenetic footprinting. Mol Cell Biol 25:3076-3086.

de Souza FS, Nasif S, López-Leal R, Levi DH, Low MJ, Rubinsten M (2011) The estrogen receptor $\alpha$ colocalizes with proopiomelanocortin in hypothalamic neurons and binds to a conserved motif present in the neuronspecific enhancer nPE2. Eur J Pharmacol 660:181-187.

Ehrman LA, Mu X, Waclaw RR, Yoshida Y, Vorhees CV, Klein WH, Campbell $\mathrm{K}$ (2013) The LIM homeobox gene Isll is required for the correct development of the striatonigral pathway in the mouse. Proc Natl Acad Sci USA 110:E4026-E4035.

Farooqi IS, Yeo GS, Keogh JM, Aminian S, Jebb SA, Butler G, Cheetham T, O'Rahilly S (2000) Dominant and recessive inheritance of morbid obesity associated with melanocortin 4 receptor deficiency. J Clin Invest 106: 271-279.

Franchini LF, López-Leal R, Nasif S, Beati P, Gelman DM, Low MJ, de Souza FJ, Rubinstein M (2011) Convergent evolution of two mammalian neu- ronal enhancers by sequential exaptation of unrelated retroposons. Proc Natl Acad Sci USA 108:15270-15275.

Gaulton KJ, Ferreira T, Lee Y, Raimondo A, Mägi R, Reschen ME, Mahajan A, Locke A, Rayner NW, Robertson N, Scott RA, Prokopenko I, Scott LJ, Green T, Sparso T, Thuillier D, Yengo L, Grallert H, Wahl S, Frånberg M, et al. (2015) Genetic fine mapping and genomic annotation defines causal mechanisms at type 2 diabetes susceptibility loci. Nat Genet 47:1415-1425.

Hayashi S, McMahon AP (2002) Efficient recombination in diverse tissues by a tamoxifen-inducible form of cre: a tool for temporally regulated gene activation/inactivation in the mouse. Dev Biol 244:305-318.

Heisler LK, Cowley MA, Tecott LH, Fan W, Low MJ, Smart JL, Rubinstein M, Tatro JB, Marcus JN, Holstege H, Lee CE, Cone RD, Elmquist JK (2002) Activation of central melanocortin pathways by fenfluramine. Science 297:609-611

Hixson JE, Almasy L, Cole S, Birnbaum S, Mitchell BD, Mahaney MC, Stern MP, MacCluer JW, Blangero J, Comuzzie AG (1999) Normal variation in leptin levels is associated with polymorphisms in the proopiomelanocortin gene, POMC. J Clin Endocrinol Metab 84:3187-3191.

Huszar D, Lynch CA, Fairchild-Huntress V, Dunmore JH, Fang Q, Berkemeier LR, Gu W, Kesterson RA, Boston BA, Cone RD, Smith FJ, Campfield LA, Burn P, Lee F (1997) Targeted disruption of the melanocortin-4 receptor results in obesity in mice. Cell 88:131-141.

Ibrahim N, Bosch MA, Smart JL, Qiu J, Rubinstein M, Rønnekleiv OK, Low MJ, Kelly MJ (2003) Hypothalamic proopiomelanocortin neurons are glucose responsive and express K+ ATP channels. Endocrinology 144: 1331-1340.

Jeong JH, Lee DK, Jo YH (2017) Cholinergic neurons in the dorsomedial hypothalamus regulate food intake. Mol Metab 6:306-312.

Jeong JH, Lee DK, Liu SM, Chua SC Jr, Schwartz GJ, Jo YH (2018) Activation of temperature-sensitive TRPV1-like receptors in ARC POMC neurons reduces food intake. PLoS Biol 16:e2004399.

Japón MA, Rubinstein M, Low MJ (1994) In situ hybridization analysis of anterior pituitary hormone gene expression during fetal mouse development. J Histochem Cytochem 42:1117-1125.

Kimura S, Hara Y, Pineau T, Fernandez-Salguero P, Fox CH, Ward JM, Gonzalez FJ (1996) The T/ebp null mouse: thyroid-specific enhancerbinding protein is essential for the organogenesis of the thyroid, lung, ventral forebrain, and pituitary. Genes Dev 10:60-69.

Krude H, Biebermann H, Luck W, Horn R, Brabant G, Grüters A (1998) Severe early-onset obesity, adrenal insufficiency and red hair pigmentation caused by POMC mutations in humans. Nat Genet 19:155-157.

Kusakabe T, Kawaguchi A, Hoshi N, Kawaguchi R, Hoshi S, Kimura S (2006) Thyroid-specific enhancer-binding Protein/NKX2.1 is required for the maintenance of ordered architecture and function of the differentiated thyroid. Mol Endocrinol 20:1796-1809.

Lam DD, de Souza FS, Nasif S, Yamashita M, López-Leal R, Otero-Corchon V, Meece K, Sampath H, Mercer AJ, Wardlaw SL, Rubinstein M, Low MJ (2015) Partially Redundant Enhancers Cooperatively Maintain Mammalian Pomc Expression Above a Critical Functional Threshold. PLoS Genet 11:e1004935.

Lee BJ, Cho GJ, Norgren RB Jr, Junier MP, Hill DF, Tapia V, Costa ME, Ojeda SR (2001) TTF-1, a homeodomain gene required for diencephalic morphogenesis, is postnatally expressed in the neuroendocrine brain in a developmentally regulated and cell-specific fashion. Mol Cell Neurosci $17: 107-126$

Loos RJ, Bouchard C (2008) FTO: the first gene contributing to common forms of human obesity. Obes Rev 9:246-250.

Marín O, Baker J, Puelles L, Rubenstein JL (2002) Patterning of the basal telencephalon and hypothalamus is essential for guidance of cortical projections. Development 129:761-773.

Mazzoni EO, Mahony S, Closser M, Morrison CA, Nedelec S, Williams DJ, An D, Gifford DK, Wichterle H (2013) Synergistic binding of transcription factors to cell-specific enhancers programs motor neuron identity. Nat Neurosci 16:1219-1227.

Merkle FT, Maroof A, Wataya T, Sasai Y, Studer L, Eggan K, Schier AF (2015) Generation of neuropeptidergic hypothalamic neurons from human pluripotent stem cells. Development 142:633-643.

Miccadei S, De Leo R, Zammarchi E, Natali PG, Civitareale D (2002) The synergistic activity of thyroid transcription factor 1 and pax 8 relies on the Promoter/Enhancer interplay. Mol Endocrinol 16:837-846.

Nasif S, de Souza FS, González LE, Yamashita M, Orquera DP, Low MJ, 
Rubinstein M (2015) Islet 1 specifies the identity of hypothalamic melanocortin neurons and is critical for normal food intake and adiposity in adulthood. Proc Natl Acad Sci USA 112:E1861-E1870.

Orquera DP, Nasif S, Low MJ, Rubinstein M, de Souza FSJ (2016) Essential function of the transcription factor rax in the early patterning of the mammalian hypothalamus. Dev Biol 416:212-224.

Padilla SL, Carmody JS, Zeltser LM (2010) Pomc-expressing progenitors give rise to antagonistic neuronal populations in hypothalamic feeding circuits. Nat Med 16:403-405.

Pinheiro J, Bates D, DebRoy S, Sarkar D and R Core Team (2018) nlme: linear and nonlinear mixed effects models. R package version 3.1-137. Vienna, Austria: R Foundation.

Rau AR, Hentges ST (2017) The relevance of AgRP neuron-derived GABA inputs to POMC neurons differs for spontaneous and evoked release. J Neurosci 37:7362-7372.

Rotimi CN, Comuzzie AG, Lowe WL, Luke A, Blangero J, Cooper RS (1999) The quantitative trait locus on chromosome 2 for serum leptin levels is confirmed in African-Americans. Diabetes 48:643-644.

Rubinstein M, Low MJ (2017) Molecular and functional genetics of the proopiomelanocortin gene, food intake regulation and obesity. FEBS Lett 591:2593-2606.

Santangelo AM, de Souza FS, Franchini LF, Bumaschny VF, Low MJ, Rubinstein M (2007) Ancient exaptation of a CORE-SINE retroposon into a highly conserved mammalian neuronal enhancer of the proopiomelanocortin gene. PLoS Genet 3:1813-1826.

Sanz E, Quintana A, Deem JD, Steiner RA, Palmiter RD, McKnight GS (2015) Fertility-regulating Kiss1 neurons arise from hypothalamic POMC-expressing progenitors. J Neurosci 35:5549-5556.

Scuteri A, Sanna S, Chen WM, Uda M, Albai G, Strait J, Najjar S, Nagaraja R, Orrú M, Usala G, Dei M, Lai S, Maschio A, Busonero F, Mulas A, Ehret GB, Fink AA, Weder AB, Cooper RS, Galan P, et al. (2007) Genomewide association scan shows genetic variants in the FTO gene are associated with obesity-related traits. PLoS Genet 3:e115.

Smemo S, Tena JJ, Kim KH, Gamazon ER, Sakabe NJ, Gómez-Marín C, Aneas I, Credidio FL, Sobreira DR, Wasserman NF, Lee JH, Puviindran V, Tam D, Shen M, Son JE, Vakili NA, Sung HK, Naranjo S, Acemel RD, Manzanares M, et al. (2014) Obesity-associated variants within FTO form long-range functional connections with IRX3. Nature 507:371-375.

Sussel L, Marin O, Kimura S, Rubenstein JL (1999) Loss of Nkx2.1 homeobox gene function results in a ventral to dorsal molecular respecification within the basal telencephalon: evidence for a transformation of the pallidum into the striatum. Development 126:3359-3370.

Thorleifsson G, Walters GB, Gudbjartsson DF, Steinthorsdottir V, Sulem P, Helgadottir A, Styrkarsdottir U, Gretarsdottir S, Thorlacius S, Jonsdottir I, Jonsdottir T, Olafsdottir EJ, Olafsdottir GH, Jonsson T, Jonsson F, Borch-Johnsen K, Hansen T, Andersen G, Jorgensen T, Lauritzen T, et al. (2009) Genome-wide association yields new sequence variants at seven loci that associate with measures of obesity. Nat Genet 41:18-24.

Veneziano L, Parkinson MH, Mantuano E, Frontali M, Bhatia KP, Giunti P (2014) A novel de novo mutation of the TITF1/NKX2-1 gene causing ataxia, benign hereditary chorea, hypothyroidism and a pituitary mass in a UK family and review of the literature. Cerebellum 13:588-595.

Wang L, Meece K, Williams DJ, Lo KA, Zimmer M, Heinrich G, Martin Carli J, Leduc CA, Sun L, Zeltser LM, Freeby M, Goland R, Tsang SH, Wardlaw SL, Egli D, Leibel RL (2015) Differentiation of hypothalamic-like neurons from human pluripotent stem cells. J Clin Invest 125:796-808.

Wheeler E, Huang N, Bochukova EG, Keogh JM, Lindsay S, Garg S, Henning E, Blackburn H, Loos RJ, Wareham NJ, O’Rahilly S, Hurles ME, Barroso I, Farooqi IS (2013) Genome-wide SNP and CNV analysis identifies common and low-frequency variants associated with severe early-onset obesity. Nat Genet 45:513-517.

Xu AW, Kaelin CB, Takeda K, Akira S, Schwartz MW, Barsh GS (2005) PI3K integrates the action of insulin and leptin on hypothalamic neurons. 115:951-958.

Yang L, Cai CL, Lin L, Qyang Y, Chung C, Monteiro RM, Mummery CL, Fishman GI, Cogen A, Evans S (2006) IsllCre reveals a common bmp pathway in heart and limb development. Development 133:15751585.

Yaswen L, Diehl N, Brennan MB, Hochgeschwender U (1999) Obesity in the mouse model of pro-opiomelanocortin deficiency responds to peripheral melanocortin. Nat Med 5:1066-1070.

Yee CL, Wang Y, Anderson S, Ekker M, Rubenstein JL (2009) Arcuate nucleus expression of NKX2.1 and DLX and lineages expressing these transcription factors in neuropeptide $\mathrm{Y}+$, proopiomelanocortin + , and tyrosine hydroxylase + neurons in neonatal and adult mice. J Comp Neurol 517:37-50.

Young JI, Otero V, Cerdán MG, Falzone TL, Chan EC, Low MJ, Rubinstein M (1998) Authentic cell-specific and developmentally regulated expression of pro-opiomelanocortin genomic fragments in hypothalamic and hindbrain neurons of transgenic mice. J Neurosci 18:6631-6640. 